\title{
Grebe/Bachems photometrische Analyse der Linienprofile und die Gravitations-Rotverschiebung: 1919 bis 1922
}

\author{
Klaus Hentschel \\ Institut für Wissenschaftsgeschichte, Universität Göttingen, \\ Humboldtallee 11, D-3400 Göttingen, Germany
}

Received 31 January 1991

\begin{abstract}
Summary
An effort of proponents of relativity theory to find evidence for the so-called gravitational red-shift of spectral lines as one of the experimental consequences of Einstein's generalized theory of relativity is reconsidered with reference to hitherto unpublished documents. It is shown how much interest Albert Einstein in fact took, around 1920, in the data analysis of Leonhard Grebe and Albert Bachem, who tried to explain why most earlier efforts to find the gravitational red-shift had failed. They carefully measured the line profiles of the spectral lines both in the sun's Fraunhofer lines and in pressure-independent laboratory comparison spectra. Then they rejected all lines with strong neighbouring lines which could cause apparent shifts of the line centres; the remaining seven well-isolated lines showed the gravitational red-shift with acceptable accuracy. Nevertheless, their claim to have successfully isolated the relativistic effect never convinced the astrophysicists of their day-the reasons for the scientific community's scepticism, contrasted against the enthusiastic group of Einstein sympathizers, are also discussed.
\end{abstract}

\section{Contents}

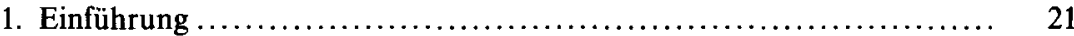

2. Forschungsförderung um 1920 am Beispiel der Unterstützung Grebe/Bachems durch Einstein ................................. 22

3. Details der photometrischen Arbeiten von Grebe und Bachem .......... 27

4. Statistischer Ausbau der Resultate durch Grebe ................... 31

5. Bewertung der Ergebnisse Bachem und Grebes durch Dritte .......... 33

6. Das weitere Schicksal Bachems und Grebes ...................... 42

7. Literaturverzeichnis............................................... 43

\section{Einführung}

In diesem Aufsatz geht es um den Anfang der zwanziger Jahre heftig diskutierten Versuch zweier Bonner Physiker, eine Erklärung des Versagens der Experimente vor 1920 zu liefern, die zur Prüfung der von Albert Einstein seit 1907 immer wieder gemachten Voraussage einer geringfügigen Verschiebung der Absorptionslinien im Sonnenspektrum hin zum roten Ende des Spektrums relativ zu irdischen Vergleichsspektren angestellt worden waren, ohne eindeutige Ergebnisse zu bringen. ${ }^{1}$

Nach der sensationellen Bestätigung der Lichtablenkung im Schwerefeld der Sonne während der Sonnenfinsternis 1919 und der zufriedenstellenden Übereinstimmung zwischen Theorie und astronomischen Beobachtungen in Bezug auf die Bewegung des Merkurperihels lag Einstein zunehmend an einer Klärung der noch offenen Frage, ob

\footnotetext{
${ }^{1}$ Für Übersichtsartikel vgl. Earman/Glymour [1980] (Theorie) und Forbes [1961] (Experimente).
} 
auch die Rotverschiebung im Gravitationsfeld (im folgenden abgekürzt GRV) sich im Spektrum der Sonne bestätigen läßt. Nur war im Unterschied zur Lichtablenkung und zur Merkurperiheldrehung im Falle der Rotverschiebung der Spektrallinien die experimentelle Situation sehr viel unübersichtlicher, denn auch Druckeinflüsse und Bewegungen der leuchtenden Gase der Sonne konnten zu Spektrallinienverschiebungen in gleicher Höhe führen. Unter denjenigen, die bereits vor $1920 \mathrm{MeBreihen} \mathrm{zur}$ Untersuchung der Verschiebung von Spektrallinien unternommen hatten und dabei die erforderliche Genauigkeit von $10^{-6}$ erreicht hatten (Schwarzschild in Potsdam, Adams u. St. John am Mt. Wilson Solar Observatory, Evershed am Kodaikanal Observatory, Madras sowie Fabry u. Buisson in Marseille) waren ferner noch etliche instrumentelle Details heftig umstritten, darunter auch die Kriterien der Auswahl der überhaupt zu untersuchenden und in die statistische Auswertung einzubeziehenden Linien. Im einzelnen war z.B. ungeklärt: welche Linien Temperatur- und druckabhängige Verschiebungen zeigen, die vor dem Vergleich herausgerechnet werden müssen, ${ }^{2}$ ob, und wenn ja welche Teile der Sonne, Konvektionsströme aufweisen und von welcher Größenordnung die relativen Geschwindigkeiten sind, die als überlagerter Doppler-RV vor dem Vergleich von Sonnen- und Erdlinien herausgerechnet werden müssen. Ferner war unklar, ob metallische oder nicht-metallische Linien für Tests geeigneter seien, bzw. ob unter den metallischen z.B. die sehr starken und gut vermessenen Eisen- oder Magnesiumlinien oder feinere, aber sehr viel lichtschwächere Linien ausgewählt werden sollten. Bei der Auswahl der Linien wurde von den Experimentatoren eine jeweils andere Mischung der Kriterien: Intensität, Feinheit, Symmetrie oder/und die Isoliertheit von anderen Linien als vorrangig betrachtet, und es war z.B. nicht klar, inwiefern benachbarte Linien im Spektrum durch Superpositionseffekte (unsymmetrische Anlehnung an starke Metallinien) zu Reduzierung des Eignungsgrades führten, oder ob nur die Verschiebung der Linienbanden verschiedener Elemente variieren oder auch innerhalb der Banden eines Elementes Schwankungen der Verschiebung auftreten. ${ }^{3}$ SchlieBlich war selbst die Zuordnung einiger problematischer Linien zu den erzeugenden Elementen noch ungeklärt (insb. das sog. Cyanbandenproblem). ${ }^{4}$

Um 1920 begannen sich zwar die Kriterien für die Auswahl von zur Messung brauchbarer Linien zu festigen, gefördert durch die Bemühungen der Standardkommissionen (insb. der International Union for Co-Operation in Solar Research bzw. ab 1919 von deren Nachfolgeorganisation, der International Astronomical Union), die auf eine standardisierte und immer genauere Bestimmung der Wellenlängen in Sonnen- und irdischen Spektren hinarbeiteten, ${ }^{5}$ doch noch immer standen sich positive und negative Aussagen zum Vorhandensein der GRV Einsteins unvermittelt entgegen.

\section{Forschungsförderung um 1920 am Beispiel der Unterstützung Grebe/Bachems durch Einstein}

Vor diesem Hintergrund einer experimentell völlig uneinheitlichen und für den Nicht-Fachmann unüberschaubaren Situation muß unsere Fallstudie gesehen werden. Die meisten gegen die GRV sprechenden Daten waren im Ausland gewonnen worden.

\footnotetext{
${ }^{2}$ So wurde z.B. die Cyanbande lange Zeit für druckunabhängig gehalten, bis King [1921] deren Druckabhängigkeit zeigte--vgl. Bachem [1920], v. Laue [1924].

${ }^{3}$ Siehe dazu Schwarzschild [1914], Evershed [1918]a, St. John [1917]b, King [1921].

${ }^{4}$ Siehe Schwarzschild [1914], Grotian u. Runge [1914], Barratt [1920], Bachem [1920], Birge [1922].

${ }^{5}$ Auf die vielfältigen und interessanten Querbezüge zum Meßwesen und zur Definition des Meters in Vielfachen der roten $\mathrm{Cd}$-Linie kann ich hier nicht eingehen.
} 


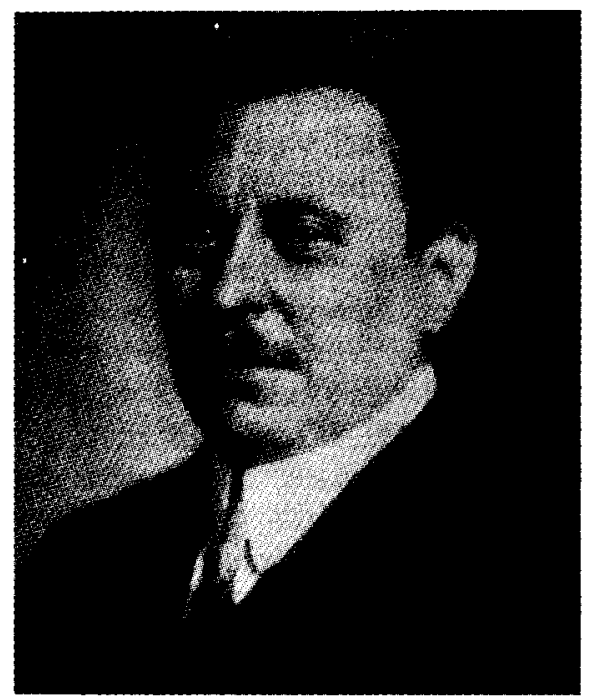

Abbildung 1. Leonhard Grebe (1883-1967): Photo aus: Reichshandbuch der Deutschen Gesellschaft, Bd. 1 (1930), S. 586-587.

Die beiden wichtigsten Orte in Deutschland, in denen spektroskopische Präzisionsmessung schwerpunktmäßig betrieben wurden, waren Bonn ${ }^{6}$ und Potsdam, ${ }^{7}$ und so ist es nicht allzu erstaunlich, daß Einstein, der seit seinem Wechsel nach Berlin erheblichen forschungspolitischen Einfluß hatte, den Bonner Forschern Gelder der nach der Niederlage Deutschlands im 1. Weltkrieg und der dadurch entstandenen katastrophalen Wirtschaftslage gegründeten Notgemeinschaft der Deutschen Wissenschaft sowie des Kaiser-Wilhelm-Instituts für Physik für Forschungen über die RV am Physikalischen Institut der Universität Bonn zukommen ließ.8

Am Physikalischen Institut der Universität Bonn interessierten sich insb. die beiden Physiker ${ }^{9}$ Albert Bachem (1888-1957) und Leonhard (Christian Friedrich) Grebe (1883-1967) für die Möglichkeit, zur Verbesserung des Wissens um die Rotverschiebung von Spektrallinien beizutragen, und zwar mit der Grundidee, in den alten Daten anderer Experimentatoren durch rigide Selektion 'geeigneter' Linien eine Bestätigung der GRV-Voraussage der ART zu bekommen. Wie sie mit Einstein in Kontakt gekommen sind, läßt sich aus den erhaltenen Dokumenten nicht mehr rekonstruieren, aber sie müssen mit Einstein bereits im Jahr 1918 über eine eventuelle Unterstützung durch die Notgemeinschaft verhandelt haben. Am 26 Januar 1919 bedankten sich die beiden jedenfalls für eine ihnen bewilligte 'Arbeitsbeihilfe' und 'die so liebenswürdige Förderung, die Sie [Einstein] unserer Arbeit angedeihen lassen'. ${ }^{10}$

\footnotetext{
${ }^{6}$ Seit den Untersuchungen von Heinrich Kayser (1853-1940), dem Doyen der Spektroskopie in Deutschland; vgl. Kayser/Eversheim [1913] zur instrumentellen Ausstattung des dortigen Instituts.

${ }^{7}$ Seit den Untersuchungen Hermann Carl Vogels (1841-1907) über Doppler-Verschiebungen in Sternspektren am Astrophysikalischen Observatorium Potsdam.

${ }^{8}$ Für eine Analyse der Förderung durch die Notgemeinschaft siehe Nipperday/Schmugge [1970], Richter [1971]. Zum Kaiser-Wilhelm Institut für Physik, das erst in den 30er Jahren ein eigenes Gebäude erhielt, siehe z.B. Heisenberg [1971], Vierhaus u. vom Brocke [1990]. Zur 'Not der deutschen Wissenschaft' in dieser Zeit vgl. auch die Internationale Monatsschrift für Wissenschaft, Kunst und Technik, 15 [1920-21], Heft 1, 5, 7.

${ }^{9}$ Vgl. J. C. Poggendorffs biographisch-literarisches Handwörterbuch, Bände V-VIIb.

${ }^{10}$ Bachem u. Grebe an Einstein, 26. Januar 1919, Collected Papers of Albert Einstein (im folgenden abgekürzt: CPAE), Sign. 6 046-1.
} 
Ferner erhielten sie, wiederum aufgrund der Fürsprache Einsteins, vom Kuratorium des Kaiser-Wilhelm-Instituts für Physik, noch im gleichen Jahr 2000 Mark für ihre spektroskopischen Untersuchungen. ${ }^{11}$

Ursprünglich war dieses Geld für den Kauf eines Mikrophotometers bestimmt, d.h. eines Apparates, mit dem sog. Linienprofile, also die Anhängigkeit der Intensität von der Wellenlänge, exakt aufgezeichnet werden konnten, indem die Schwärzungsgrade der Photoplatte analog über eine Photozelle in einen elektrischen Strom umgesetzt wurden. ${ }^{12}$ Doch auch der am Astrophysikalischen Observatorium in Potsdam arbeitende Astronom Erwin Finlay Freundlich ${ }^{13}$ (1885-1964) hatte gerade eines dieser begehrten Instrumente gekauft, so daß es sich anbot, daß Bachem und Grebe ihre Messungen in Potsdam durchführten. Wie z.B. aus einem Brief L.C. Glasers an Stark hervorgeht, ${ }^{14}$ bestand damals eine harte Konkurrenz der Interessenten für die Mikrophotometer der Firma Töpfer, da diese nur sehr wenige herstellte und später ganz bankrott machte. ${ }^{15}$ Der Preis eines gesamten Kochschen Mikrophotometers lag 1920 im inflationsgeplagten Deutschland um 10000-12000 Reichsmark, so daß der Kauf eines eigenen Gerätes von Grebe um 1920 zwar erwogen, aber nicht realisiert worden ist. ${ }^{16}$ Insofern war dieser Vorschlag der Nutzung eines dieser teuren Geräte von mehreren Experimentatoren aus verschiedenen Teilen Deutschlands sehr vernünftig-er antizipierte Formen der Forschungskooperation, wie sie heute, bei noch erheblich intensivierten Kosten für aufwendigste Meßapparaturen, völlig normal geworden ist. In diesem Sinne schlug Einstein den Bonner Physikern vor:

Wie diese Summe zu verwenden ist, darüber werden wir uns in der nächsten Zeit $\mathrm{zu}$ einigen haben. Ich ersuche Sie, mir bezüglich der Verwendung des Geldes einen Vorschlag zu machen. Herr Freundlich teilt mir mit, dass sein Kochsches Photometer in den nächsten Wochen montiert wird und dann Ihnen bezw. Ihrem Mitarbeiter dort zur Verfügung steht. ${ }^{17}$

Der Plan von Bachem und Grebe, im Sommer 1919 das Berliner Astrophysikalische Observatorium zu besuchen und dort am Freundlich'schen Instrument $\mathrm{zu}$ arbeiten, verzögerte sich zunächst. ${ }^{18}$

Indessen hatten die beiden auch ohne Zugang zum Berliner Photometer mit weit primitiveren Mitteln ein interessantes Resultat erhalten. Sie hatten sich einfach mit dem Vergleich stark und schwach belichteter Aufnahmen des gleichen Spektrums beholfen. Während in den schwach belichteten Aufnahmen nur die Intensitätsmaxima eine Schwärzung der Photoplatte herbeiführten, wurde bei lange belichteten Aufnahmen auch der schwache Teil einer Spektrallinie in vollkommene Schwärzung umgesetzt. Die Versetzungen der dünnen Linien der schwach belichteten Aufnahmen gegenüber der

\footnotetext{
${ }^{11}$ Vgl. Einstein an das Kuratorium des Kaiser-Wilhelm Instituts für Physik, 25 April 1919, Archiv der Max-Planck-Gesellschaft, Berlin-Dahlem (im folgenden abgekürzt: AMPG), Abt. I, Rep. 1A, Mappe $\mathrm{Nr}$ 1656, Dok. Nr. 55-56 sowie den Kassenauszug vom 27. Juni 1919, ibid., Dok. Nr. 69.

${ }^{12} \mathrm{Vgl}$. zur Funktionsweise dieses damals hochmodernen Präzisionsinstrumentes: Koch [1913] Nützliche Überblicke zur Geschichte der Photometrie insb. im 19. Jahrhundert geben Herrmann u. Hoffmann [1976], Wolfschmidt [1989].

${ }^{13} \mathrm{Zu}$ Freundlich siehe: v. Klüber [1965], Forbes [1982] und Hentschel [1992].

${ }^{14}$ Stiftung Preußischer Kulturbesitz (SPK), Nachlaß Stark, Mappe Glaser, zu Glaser siehe unter Fußnote 53 .

${ }^{15}$ Siehe Grebe an Debye, 10. März 1924, AMPG, Abt. 1, Rep. 34, Mappe 3.

${ }^{16} \mathrm{Vgl}$. Grebe an Einstein, 6. Juni 1919, AMPG, Abt. 1, Rep. 34, Mappe 3 sowie Grebe an Einstein, 12. Juli 1920, CPAE, Sign. 6 048. Zum Vergleich: im Jahr 1920 erhielt die Notgemeinschaft insg. 20 Millionen Mark Reichszuschüsse.

${ }^{17}$ Einstein an Grebe, 26. April 1920, AMPG, Abt. I, Rep. 34, Mappe Nr. 3.

${ }^{18} \mathrm{Vgl}$. Grebe an Einstein, 6. Juni 1919, AMPG, Abt. 1, Rep. 34, Mappe 3.
} 
Mitte der breiten Streifen in langbelichteten Platten ließ dann auf eine Asymmetrie der jeweiligen Linie schließen. Weil sich solche Unsymmetrien aber verkleinernd auf den in der Sonne vorhandenen Verschiebungseffekt auswirken konnten, könnte dies erklären, warum Sonnenlinien im Vergleich mit irdischen Lichtquellen 'zu klein gemessen werden in Übereinstimmung mit den Messungen von Schwarzschild, St. John und uns. Gleichzeitig müssen diese Abweichungen verschieden herauskommen je nach der Struktur der einzelnen Linien, wie es ebenfalls durch das Experiment bestätigt wird. ${ }^{19}$

Darum konnten sie in ihrer ersten Arbeit über die RV der Spektrallinien immerhin feststellen, 'daß eine Verschiebung vorhanden ist [...], daß aber eine Mittelbildung über die gemessenen Linien nicht erlaubt ist, weil die Verschiebung für die einzelnen Linien weit über die Messungsfehler variiert, so da 3 also die einzelnen Linien eine reelle Verschiedenheit in ihrer Abweichung aufweisen'. ${ }^{20}$ Wenn dem so war, so konnte nur eine photometrische Auswertung der einzelnen Linienprofile (anstelle der summarischen Mittelung über viele Linien) genauen Aufschluß über die tatsächliche RV geben. Einstein zeigte sich angetan von dem Resumé Grebes, 'daß der Schwarzschildsche und St. Johnsche Schluß, daß der theoretische Effekt nicht vorhanden ist jedenfalls nicht sichergestellt ist, wenn wir auch das Gegenteil noch nicht eindeutig beweisen können.'21

Durch die von Einstein lancierte Förderung wurden Bachem und Grebe in die Lage versetzt, im Frühjahr 1920 am besser ausgerüsteten Potsdamer Observatorium auch die in Bonn bis dato erst nicht möglichen und dann wegen eines Schraubenfehlers mißglückten ${ }^{22}$ photometrischen Verschiebungsmessungen durchzuführen, die ihre groben photographischen Plattenvergleiche präzisieren konnten. ${ }^{23}$ Der früher erhaltene Zuschuß von 2000 Mark wurde zur Finanzierung ihres Aufenthaltes in Potsdam verbraucht, da wegen der zunehmenden Inflationsrate die Gefahr der restlosen Entwertung bestand. ${ }^{24}$

Einstein war von dem Gedanken der beiden Bonner Physiker, daß bislang unerkannte Asymmetrien der Grund für die bisher ausgebliebene Bestätigung der GRV sein könnten, offenbar so angetan, daß er den beiden noch vor Ausführung der Experimente in Potsdam bereits zusicherte, für den faksimilierten Abdruck der Photogramme sorgen zu wollen. ${ }^{25}$ Einem seiner Briefe an Grebe fügte er ein bemerkenswertes Postskriptum bei, offenbar besorgt um die rasche Umstimmung der noch sehr skeptischen scientific community:

Wann werden Sie das Beobachtungsmaterial zu Ihrer Arbeit über die Cyanbande der Öffentlichkeit zugänglich machen? Ohne eine solche Veröffentlichung werden die Fachgenossen Ihrer Arbeit kaum irgend welche Beweiskraft zuerkennen. ${ }^{26}$

${ }^{19}$ Grebe an Einstein, 6. Juni 1919, AMPG, 1. Abt. Rep. 34, Mappe 3.

${ }^{20}$ Grebe/Bachem [1919] S. 456.

${ }^{21}$ Grebe an Einstein, 6. Juni 1919, AMPG, Abt. 1, Rep. 34, Mappe 3.

${ }^{22}$ Siehe Grebe an Einstein, 23. December 1919, AMPG, Abt. 1, Rep. 34, Mappe 3.

${ }^{23} \mathrm{Vgl}$. dazu den Brief Bachem/Grebes an Einstein, 26. Januar 1919, CPAE, Sign. 6 046-1 und Grebe/Bachem [1920]a S. 51.

${ }^{24}$ Siehe Grebe an Debye, 10. März 1924, AMPG, Abt. 1, Rep. 34, Mappe 3. Viele der teilweise geradezu hektischen Geräteankäufe für Forschungszwecke um 1920 sind aus dieser Situation heraus zu verstehen, daB man bestekende monetäre Guthaben und Rücklagen sinnvoll einsetzen wollte, bevor sie durch die Inflation entwertet wurden.

${ }^{25} \mathrm{Vgl}$. Grebe an AE, 26 Januar 1919: 'Die Veröffentlichung der Photogramme, die Sie uns ebenfalls so freundlich sein wollen zu ermöglichen, begrüßen wir natürlich mit lebhaftester Freude'.

${ }^{26}$ Einstein an Grebe, 9. Juli 1920, AMPG, Abt. 1, Rep. 34, Mappe 3-vgl. auch Grebe an Einstein, 12. Juli 1920, CPAE, Sign. 6048. 
Aus der von Einstein 1920 ebenfalls ins Auge gefaßten Publikation der Resultate in den Sitzungsberichten der Preußischen Akademie der Wissenschaften, Berlin, wurde nur auf Grund einer technisch bedingten Verzögerung in der Herstellung der Mikrophotogramme nichts. ${ }^{27}$ Ihre ersten Aufnahmen waren nämlich nicht gelungen, weil die Kaliumzelle des Mikrophotometers zu träge reagierte, wodurch die Messungen nicht mehr die erforderliche Genauigkeit von 1/1000 $\AA$ hatten. Doch das Problem der Trägheit der photoelektrischen Zellen in den Photometern Kochscher Bauart wurde gerade in dieser Zeit durch Neukonstruktionen des registrierenden Mikrophotometers durch Fritz Goos u.a. gelöst. ${ }^{28}$ Hieran zeigt sich, wie technologische Entwicklungen (hier die von Photozellen) eng verzahnt sind mit bestimmten Experimenten der Grundlagenforschung, durch die ein Bedarf nach Verbesserung der bestehenden Technologien erzeugt wird.

Auch im Tätigkeitsbericht des Kaiser-Wilhelm Instituts für Physik, verfaßt von seinem damals einzigen wissenschaftlichen Mitarbeiter, Erwin F. Freundlich, für den Zeitraum 1. April 1919-31. März 1920 findet diese Kooperation zwischen den Bonner Physikern und dem Berliner Mitarbeiter Einsteins ihren Niederschlag. Freundlich vergißt nicht, seine den Besuch Grebes und Bachems vorbereitenden Arbeiten 'am Koch'schen Mikrophotometer zu erwähnen, welche zum Gelingen der bemerkenswerten Untersuchungen von Grebe und Bachem über die Rot-Verschiebung der Spektrallinien erheblich beigetragen haben'. ${ }^{29}$ Der sich nach dem Abschluß der MeBreihen abzeichnende Erfolg hat viele Väter.

Nun mußten Grebe und Bachem möglichst schnell ihre aufgrund verbesserter Photozellen erhaltenen Resultate allgemein bekannt machen. Sie publizierten vorwiegend in der neugegründeten Zeitschrift für Physik, die sehr viel schneller redigierte, so daß das Risiko vermindert wurde, daß ihnen noch jemand mit ähnlichen Studien zuvorkam. Seiner Zusendung einer Durchschrift ihres ersten Aufsatzes, der dort 1920 erschien, fügte Grebe folgenden Kommentar über ihr wichtigstes Ergebnis bei:

Das Resultat scheint uns einwandfrei die Existenz des von Ihrer Theorie geforderten Verschiebungseffektes darzutun. Mit der in unserer ersten Arbeit [1919] ausgesprochenen Vermutung, daß Unsymmetrien der Linien die kleinere Verschiebung der früheren Untersuchungen verursachen, stimmen diese Ergebnisse vollkommen überein und bringen außerdem die Erklärung dieser Unsymmetrien durch Begleiter [d.h. durch störende Nebenlinien]. Die Tatsache, daß alle früheren Untersuchungen zu kleine Verschiebungswerte geben mußten, ergibt sich überraschend einfach dadurch, daß in der Zuordnung zusammengehöriger Linien durch Rowland, dessen Tabellen auch von uns wie von allen anderen Beobachtern benutzt sind, schon ein Auswahlprinzip im Sinne einer Nullverschiebung steckt, da Rowland von einem Verschiebungseffekt nichts

\footnotetext{
${ }^{27}$ Vgl. dazu den Brief Grebe/Bachems an Einstein, 18. Juni 1920, CPAE, Sign. 6 047-1.

${ }^{28} \mathrm{Zu}$ den Mikrophotometern älterer Bauart vgl. Hartmann [1899], Koch [1912], [1913], Lehmann [1913]; zu den Neukonstruktionen siehe insb. Elster u. Geitel [1912], [1920], Goos [1921] S. 313: 'Bei der neuen Zelle bedeckt der mit Kalium überzogene Silberbelag die ganze Innenwand der Glashohlkugel mit Ausnahme einer kleinen Öffnung für den Einlaß des Lichts. Mit dieser Anordnung wird verhindert, daß sich im Innern der Zelle größere nicht versilberte Glasflächen befinden, die unvermeidlicherweise schon bei Zimmertemperatur mit einer dünnen Kaliumhaut bedeckt sind. Dadurch, daß letztere beim Belichten eine Ladung erhält, die Belichtungsänderungen nicht augenblicklich folgen kann, entsteht ja nach Elster und Geitel die Trägheit der Photozellen'. Für techn. Details vgl. Wolfschmidt [1989] S. $252 \mathrm{ff}$.

${ }^{29}$ AMPG, I. Abt., Rep. 34, Mappe 12; bzw. handschriftlicher Entwurf Freundlichs im Archiv der ehemaligen Preußischen Akademie der Wissenschaften, Berlin (im folgenden abgekürzt: AAW), Akten des Astrophysikalischen Observatoriums, Nr. 135, Jahresbericht 1919/20.
} 
wußte. Wir können hier hinzufügen, daß dieses Auswahlprinzip sich besonders störend bemerkbar machen muß, wo nur der gemessene Wellenlängenwert und nicht das charakteristische Aussehen der Linie in Sonnen- und Vergleichsspektrum als Kriterium für die Zusammengehörigkeit verwendet werden kann; und das wird besonders für die schwachen Linien gelten. Wenn also, wie mehrere Forscher behaupten, diese schwache Linien keine Verschiebung zeigen, so ist das nicht weiter verwunderlich. ${ }^{30}$

Die Förderung der Studien von Bachem und Grebe hatte sich, so mußte es nach diesem Resumé scheinen, gelohnt. Nicht nur, daß die beiden Bonner die GRV in voller Stärke nachgewiesen zu haben glaubten, sie meinten auch 'überraschend einfach' erklären zu können, wieso frühere Messungen stets zu kleine Werte geliefert hatten, womit all denen, die sich auf Schwarzschilds oder St. Johns Meßreihen von 1916 bzw. 1917 berufen hatten, der Wind aus den Segeln genommen war.

\section{Details der photometrischen Arbeiten von Grebe und Bachem}

Bachem und Grebe wählten für ihre Meßreihen die sog. Cyanbande bei $3883 \AA$ aus, bei der durch vorausgegangene Messungen anderer nahegelegt und durch einen eigenen Check bestätigt worden war, daß die Parameter Druck und elektrische Feldstärke keinen nennenswerten Einfluß auf die Lage der Linien im Spektrum haben. ${ }^{31}$ Durch Beschränkung auf die Spektrallinien einer Bande erreichten sie zudem, daß auch eventuelle Relativbewegungen (Konvektionsströme, etc.) für ihre Betrachtungen irrelevant waren,

weil die zusammengehörigen Linien einer Bande [...] nicht von verschieden Zentren emittiert werden können und etwa verschiedene Doppler-effekte zeigen dürften. ${ }^{32}$

Ferner wurden alle Linienpaare, für die im Sonnen- oder im Cyanbandenvergleichsspektrum Überlagerung mit anderen starken Nachbarlinien erfolgte oder für die die Intensität zu schwach war, um scharf messen zu können, ausgeschlossen. So verblieben nur 9 von vormals 36 Linienpaaren, die den restriktiven Bedingungen Grebe und Bachems zufolge 'genügend einwandfrei sind, um zu einer Verschiebungsmessung benutzt zu werden'. ${ }^{33}$ Die nachfolgende Abbildung der Tafel III aus Grebe/Bachem [1920] illustriert das Auswahlverfahren (Abbildung 2).

Um Mißverständnisse zu vermeiden, sei hier ausdrücklich erwähnt, daß auch andere Experimentatoren vor Grebe und Bachem bereits vor der Auswertung ihrer Messungen sorgfältige Vorauswahl der einzubeziehenden bzw. auszusondernden Linien betrieben. ${ }^{34}$ Das eigentlich Neue an der Arbeit der beiden Bonner Physiker von 1920 war, daß die mikrophotometrischen Aufnahmen der Linienprofile zusammen mit den Gründen, die jeweils zum Ausschluß bzw. zur Einbeziehung in ihre Auswertung geführt hatten, vollständig mitveröffentlicht wurden. Somit war hier den Lesern der

\footnotetext{
${ }^{30}$ Grebe an Einstein, 23. December 1919, AMPG, Abt. 1, Rep. 34, Mappe 3. Mit den Tabellen Rowlands ist Rowland [1896] gemeint. Rotverschiebungen von Fraunhoferlinien des Sonnenspektrums relativ zu irdischen Vergleichslinien publizierte erstmals Jewell [1896].

${ }^{31}$ Siehe Grotrian/Runge [1914], Schwarzschild [1914], Uhler/Patterson [1915], St. John [1917]b, Barratt [1920] sowie Bachem [1920] als gekürzter Auszug der von Bachem im Juli 1919 in Bonn vorgelegten Habilitationsschrift über die Stickstoffbande.

${ }^{32}$ Grebe/Bachem [1920]b S. 420.

${ }^{33}$ Grebe/Bachem [1920]b S. 420 .

${ }^{34}$ So z.B. Schwarzschild [1914], Evershed [1913]ff. und St. John [1917]ff.
} 
Klaus Hentschel
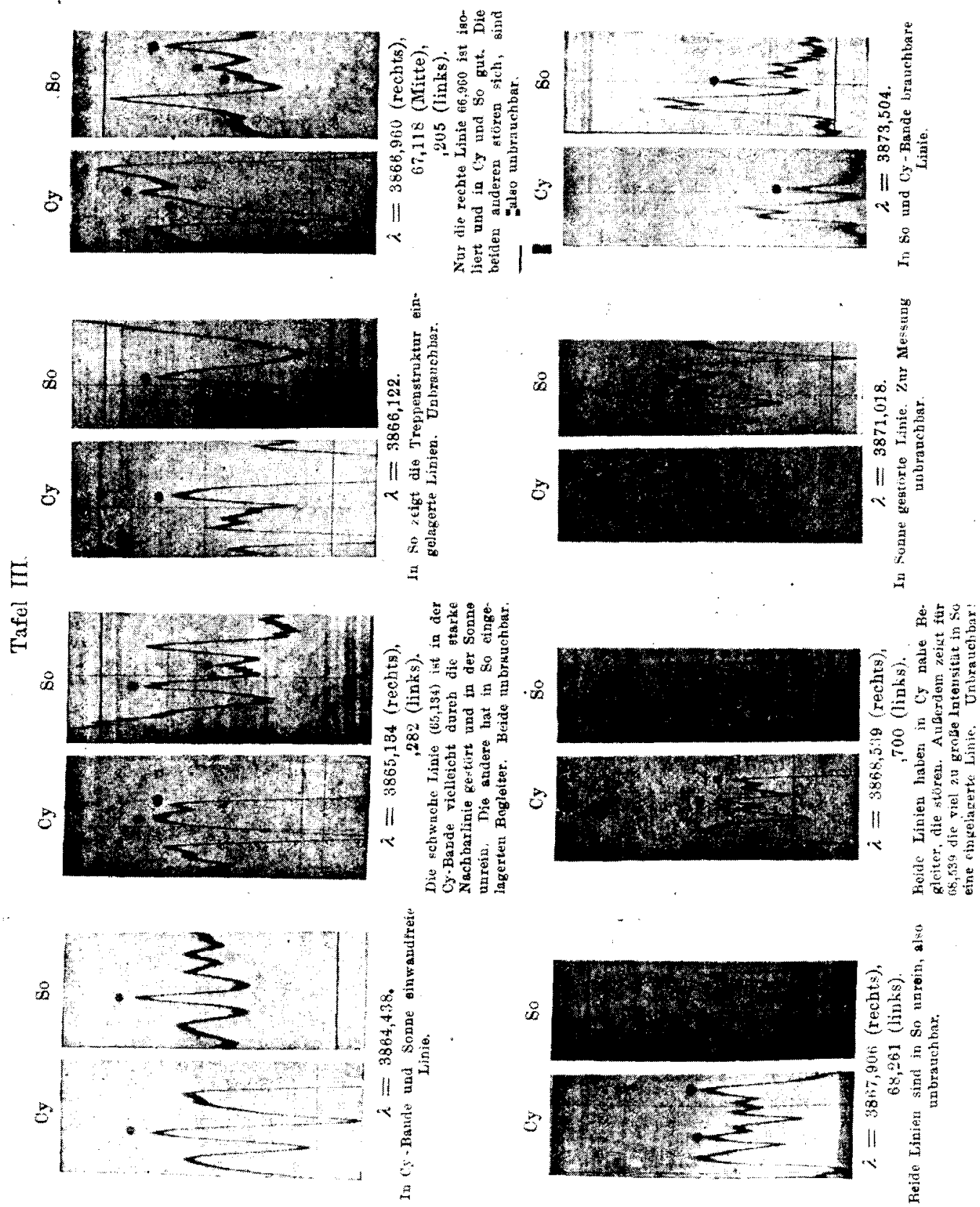

Abbildung 2. Reproduktion der Abbildung aus Grebe/Bachem [1920], in der deren Auswahlverfahren kommentiert wird. 
Publikation von 1920 erstmals die Möglichkeit einer Kontrolle über das Selektionsverfahren an die Hand gegeben-bislang mußte man sich blinden Vertrauens in die Zuverlässigkeit der Experimentatoren in deren nur summarisch erwähnte Vorauswahl fügen, wenn man nicht jede Linie selbst nachmessen wollte oder konnte. Darum hatte Einstein wie oben belegt so nachdrücklich betont, wie wichtig es sei, das Auswahlverfahren durch den kommentierten Abdruck der Linienprofile sinnfällig zu machen.

An diesem Selektionsverfahren Grebe/Bachems scheint mir ferner bemerkenswert, daß die Kriterien der Auswahl sicher nicht Bestandteil der in Rede stehenden theoretischen Alternativen (ART oder konkurrierende Erklärungen der RV) sind, sondern so restriktiv angelegt sind, daß sie systematische Fehler, gleich welchen Ursprungs, ausschließen sollen. Das hat aber zur Folge, daß der Überlappungsgrad der von Grebe/Bachem [1920] angeführten Auswahl mit früheren Messungen gering ist (mit Ausnahme ihres deutschen Vorgängers Karl Schwarzschild (1873-1916), der ebenfalls aufgrund apparativer Beschränkung nur wenige Linien betrachtete).

Tabelle 1. Anzahl der nach Grebe/Bachem geeigneten Linien der Cyanbande aus Messungen früherer Beobachter und die mittleren RV, ausgedrückt in DopplereffektÄquivalentgeschwindigkeiten, zunächst die ursprünglichen Resultate der anderen, dann die korrigierten Werte Grebe/Bachems nach Selektion.

\begin{tabular}{lcccc}
\hline \multicolumn{1}{c}{ Name } & Grebe/Bachem & Schwarzschild & St. John & Evershed/Royds \\
\hline Jahr & 1920 & 1914 & 1917 & $1914-1920$ \\
\# Linien & 11 von 36 & 7 von 33 & 5 von 43 & 2 von 12 \\
Prozent & $30 \%$ & $20^{\circ} \%$ & $12 \%$ & $16 \%$ \\
$\bar{v}$ (Orig.) $(\mathrm{km} / \mathrm{s})$ & 0.56 & 0.2 & $0.17(0.26)$ & 0.67 \\
$\bar{v}$ (korrig.) $(\mathrm{km} / \mathrm{s})$ & 0.56 & 0.63 & 0.32 & 0.67 \\
\hline
\end{tabular}

Man sieht aus Tabelle 1, daß die Einführung dieser strengen Aussonderungskriterien nicht nur in einer Einschränkung der Linien, sondern auch in einer hohen Gewichtung genau solcher Linien resultierte, die von ihren Vorgängern nicht berücksichtigt worden waren, weil diese andere Auswahlkriterien angelegt hatten. So gehen z.B. nur 2 (!) der von John Evershed (1864-1956) und Thomas Royds (1884-1955) zwischen 1914 und 1920 am Kodaikanal Observatory in Madras vermessenen Linien auch in Grebe/Bachem Statistik ein. In diesem Sinne erzeugt in dieser Phase jede Experimentatorengruppe durch die von ihr zugrunde gelegten Auswahlkriterien erst ihren Datensatz. Die geringe Überlappung dieser Materialbasis erklärt die zu dieser Zeit noch weit divergierenden Schlußfolgerungen der konkurrierenden Gruppen: deren Resultat über die Haltbarkeit oder Unhaltbarkeit der relativistischen RVPrognose ist eine Funktion der ausgewählten Linien, diese wiederum sind determiniert vom Auswahlkriterium. Zwar wird dieses Kriterium nicht über die zu testende Theorie gerechtfertigt, aber es erhebt sich natürlich gleichwohl der Verdacht, daß die Wahl der Kriterien bewußt oder unbewußt bestimmt wird von dem Bestreben der Experimentatoren, zu einer Bestätigung (oder zu einer Widerlegung) der zu testenden Theorie zu kommen. Dieser Verdacht eines wishful thinking bzw. einer allzu blauäugigen Datenauswahl bestätigt sich hier aber nicht.

Denn trotz des geringen Überlappungsgrades der Materialbasis und der völlig verschiedenen Selektionskriterien waren die von Grebe/Bachem ermittelten Rotverschiebungen (mit der Ausnahme von denen St. Johns [1917] in etwa gleich denen der anderen Gruppen und entsprachen mit der Gesamtdurchschnittsgeschwindigkeit von 
Verschiebungen der Linien der Stickstoffbande bei $3883 \AA$.

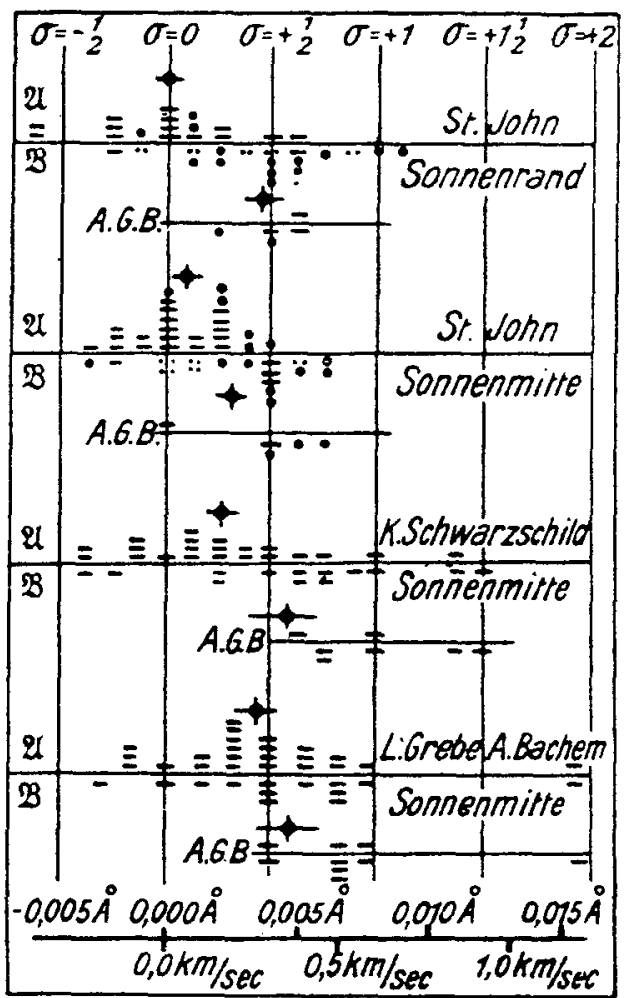

Jede Beobachtung ist durch eines der Zeichen -,,$\cdots$ angezeigt. Bei St. John bedeutet eine Beobachtung minderer Sicherheit, $\cdots$ zeigt eine Doppellinie an.

Die $\mathscr{U}$-Seite umfaßt Linien der Intensität 00 bis 1 , die $\mathfrak{B}$-Seite Linien größerer Intensität, bei St. John auch Doppellinien. - - kennzeichnet den Mittelwert; die Länge der horizontalen Arme ist dabei gleich dem mittleren Fehler.

A.G.B. $=$ Auswahl Grebe-Bachem.

Abbildung 3. Übersicht zu Verschiebungen von Spektrallinien in den Messungen von St. John, Schwarzschild und Bachem/Grebe sowie das Resultat der strengen Linienauswahl-aus Wiechert [1920]b S. 329 mit dessen originaler Bilderklärung. Die relativistische Voraussage entspricht $\sigma=1$.

$0.54 \mathrm{~km} / \mathrm{s}$ (ohne ev. Dopplerkorrektion durch Konvektionsströme) dem Wert der Einsteinprognose von $0.635 \mathrm{~km} / \mathrm{s}$ für diesen Spektralbereich immerhin schon bis auf $15 \%$. Den zeitgenössischen Stand der Beobachtungen zu Verschiebungen der Spektrallinien erfaßt sehr gut eine Abbildung in einem Artikel Emil Wiecherts (1861-1921), der um 1920 ebenso wie viele andere erklärte Gegner der Relativitätstheorie, ${ }^{35}$ deren angebliche experimentelle Bestätigungen einer strengen Prüfung unterzog.

Die vorangehende bildliche Darstellung des Auswahlverfahrens nach Wiechert [1920]b illustriert nochmals, daß der Mittelwert der Messungen für die drei Gruppen Grebe u. Bachem (Bonn), St. John (Mt. Wilson Observatory) und Schwarzschild

\footnotetext{
${ }^{35}$ So etwa Gehrcke, Riem, Glaser, Mohorovičić u.a.; zu theoretischen Alternativen der ART vgl. z.B. Hentschel [1989] Abschn. 1.5 und dort genannte Literatur.
} 
(Potsdam) von gleicher Größenordnung war, aber nicht der relativistischen Voraussage entsprach, die nur für einige Linien großer Intensität erreicht oder übertroffen wurde. Ferner fällt auf, daß für alle Beobachter die Streuung der Meßwerte, auch innerhalb der Liniengruppen großer bzw. geringer Intensität, recht beträchtlich war (von $\sigma=1$ bis zu 0 , ja bis hin zu vereinzelten Violettverschiebungen). Übrigens wertet Wiechert diese graphische Darstellung der experimentellen Resultate dahingehend aus, daß es für ihn 'ausgeschlossen' ist, 'daß auf der Sonne ein Gravitationspotential von Betrage der Einsteinschen Theorie besteht'. Hingegen schließt er einen 'geringen Gravitationseffekt' von bis zu einem Drittel der ART-GRV nicht aus. ${ }^{36}$

\section{Statistischer Ausbau der Resultate durch Grebe}

Der wesentliche Unterschied der Auswertung von Grebe/Bachem zu früheren Ausdeutungen bestand darin, daß bei ihnen die Streuung der einzelnen Verschiebungen um den Mittelwert nach der Linienselektion wesentlich geringer war. Dies führte Grebe/Bachem zu der Frage, wieso bei ihren Vorgängern (einschließlich der Meßreihe St. Johns) die dort zusätzlich in die Auswertung aufgenommenen Linienpaare mit derart großen Schwankungen behaftet waren. Ihr Ziel war es nun, dieses 'verschiedene Verhalten der verschiedenen Linien zu erklären', für das die bekannten Effekte wie Dopplereffekt oder Druckeinflüsse ja nicht herangezogen werden konnten, weil diese ja alle Linien einer Bande gleichermaßen betreffen müßten, also nicht zu unterschiedlichen Verschiebungen der Linien führen dürften.

Ihre Antwort darauf ist der Hinweis auf einen ihrer Ansicht nach in den Meßreihen ihrer Vorgänger verdeckten systematischen Fehler, der bei zu grober Selektion des Datenmaterials auftritt: ihrer Vorstellung nach entstand eine verzerrte Statistik bei früheren Experimenten dadurch, daß eine klassische Zuordnungsvorschrift (Soll-Lage der Linien im Sonnenspektrum ohne Verschiebungseffekte) im Zusammenwirken mit zufälligen Fehlern einen systematisch verzerrten Datensatz erzeuge. Die implizite Zugrundelegung von Annahmen der klassischen Theorie und der Standardverfahren zur Bestimmung von Wellenlängen erzeuge also Datenmaterial, in dem die relativistische RV bereits weggefiltert sei:

Daß die Mitbenutzung der nicht einwandfreien Spektrallinien immer zu kleine Werte für den Effekt der Gravitationsverschiebung liefert, halten wir für hinreichend dadurch erklärt, daß in allen Tabellen für die Zuordnung irdischer Emissionslinien zu den Absorptionslinien des Sonnenspektrums, die dann wieder rückwärts zur Identifizierung zusammengehöriger Linien benutzt werden, als Hauptkriterien der Zusammengehörigkeit die gleiche Lage im Spektrum benutzt worden ist, während richtig die um den Einsteinwert verschobene Lage zu benutzen wäre. Dieses falsche Auswahlprinzip bringt die durch zufällige Störungen zu stark nach Rot verschobenen Linien aus der Tabelle hinaus, während es die zu schwach verschobenen Linien darin läßt. ${ }^{37}$

Die Verschärfung der Aussonderungskriterien könne aber nur eine nachträgliche Korrektur dieses Effektes bewirken, indem dann nur von zufäligen Fehlern untangierte Linien übrigblieben, die dann einen statistisch unverfälschten Mittelwert in Übereinstimmung mit der ART lieferten. Der Nachteil dieses Verfahrens war aber, daß es zu einer sehr schlechten Statistik führte. Darum stellten beide Physiker daraufhin

\footnotetext{
${ }^{36}$ Wiechert $[1920]$ S. $326 \mathrm{f}$.

${ }^{37}$ Grebe/Bachem [1920]b S. 421.
} 
folgende Überlegung an: wenn ihre Hypothese über die Gründe für die bisherigen Mißerfolge bei der Suche nach der GRV richtig war, so müßten sich die zufälligen Fehler statistisch korrekt herausmitteln, wenn die bislang stets zugrundegelegte klassische Zuordnungsvorschrift $\left(\lambda_{\text {Soll }}=\lambda_{\text {Rowland }}\right)$ ersetzt würde durch eine von der allgemeinen Relativitätstheorie (ART) inspirierte $\left(\lambda_{\text {Soll }}=\lambda_{\text {Rowland }}+(\Delta \lambda)_{\text {ART }}\right)$ :

Wir sind der Überzeugung, daß bei der Zugrundelegung der Einsteinschen Gravitationsverschiebung als Grundwert der Linienzuordnung eine richtige Fehlergruppierung nach dem Zufall auch bei Mitbenutzung der gestörten Linien nach beiden Seiten auftreten muß. Die Prüfung dieser Auffassung muß weiterer Untersuchung vorbehalten bleiben. ${ }^{38}$

Die Ergebnisse dieser Zusatzuntersuchung, die es sich zur Aufgabe machte, 'das stark zusammengeschmolzene Beobachtungsmaterial wieder zu vergrössern', ${ }^{39}$ trug Grebe auf der wegen des Rededuells zwischen Lenard und Einstein berühmet gewordenen Bad Nauheimer Naturforscherversammlung 1920 vor-veröflentlicht wurde sie im darauffolgenden Jahr. Darin wurden 100 aufeinanderfolgende Linien der Stickstoffbande bei $3883 \AA$ aus den Rowland-Jewellschen Messungen auf ihre Verschiebung gegen das irdische Vergleichsspektrum hin untersucht, und zwar diesmal ohne jede Auswahl, denn

bei Benutzung sämtlicher Linien eines linienreichen Spektrums ohne Auswahl [muß] der richtige Verschiebungswert herauskommen; denn die Störungen werden ebensooft nach der einen wie nach der anderen Seite gehen, und diese nach dem Zufall verteilten Störungsfehler werden bei der Mittelbildung herausfallen. ${ }^{40}$

Grebes Resultat war, daß "bei Benutzung des sicher unvoreingenommenen Rowlandschen Messungsmaterials $\left[{ }^{41}\right]$ eine Rotverschiebung, wie sie von der Einsteinschen Theorie gefordert wird", vorliegt. ${ }^{42}$ Schützenhilfe erhielt Grebe durch den Marburger Theoretiker Franz Arthur Schulze (1872-1943), der dessen neue Daten einer detaillierten statistischen Analyse unterwarf, mit der quantitative Angaben über das 'Maß des Verdachtes auf systematische Fehler in Beobachtungsreihen' gemacht werden konnten. ${ }^{43}$ Dieser wahrscheinlichkeitstheoretischen Analyse zufolge war die Annahme, daß der Einsteineffekt nicht existiert, 'mit den Beobachtungen ganz unverträglich', während umgekehrt 'das tatsächliche Vorhandensein der Rotverschiebung im Sonnenspektrum in der von der Theorie verlangten Größe sehr wahrscheinlich' war. ${ }^{44}$

Mit dieser Neudeutung älteren Beobachtungsmaterials war mehr erreicht als nur eine Wiederholung früherer Behauptungen des Vorhandenseins des relativistischen Effektes, denen man, je nachdem man die Selektionskriterien der Autoren für gut

${ }^{38}$ Grebe/Bachem [1920]b S. 421f.; Hervorhebung Orig.

${ }^{39}$ Grebe [1921] S. 105.

${ }^{40}$ Ibid. (Fußnote 39). Verglichen wurden also Rowland [1896] mit Uhler/Patterson [1915].

${ }^{41}$ D.h. nach Umrechnung der Rowlandschen Werte aus Rowland [1896] in das internationale Wellenlängensystem, gemäß dem die Vergleichswerte für irdische Emissionsspektren in Uhler/Patterson [1915] vermessen worden waren. Rowlands Tabellenwerk geht zurück auf Meßreihen angestellt zusammen mit Jewell u.a. zwischen 1885 und 1895, also 30 Jahre vor Formulierung der ART und 20 Jahre vor der Entwicklung des Äquivalenzprinzips.

${ }^{42}$ Grebe [1921] S. 105.

${ }^{43}$ Den mathematischen Hintergrund hierfür bildeten Arbeiten von Franz Richarz, Ernst Neumann und F. R. Helmert, alle zit. in Schulze [1921].

${ }^{44}$ Schulze [1921] S. 373. 
befand oder nicht, zustimmen konnte oder nicht - erstmals war ein Verfahren vorgeschlagen worden, wie man aus vorliegenden Datensätzen konsistente Mittelwerte erhalten könne, und es war auch eine plausibele Erklärung dafür vorgeschlagen worden, wieso andere Experimentatoren mit ihren Daten an einer Bestätigung des 'Einsteineffektes' gescheitert waren.

\section{Bewertung der Ergebnisse Bachem und Grebes durch Dritte}

Einstein war offenbar sehr angetan von Grebe/Bachems Resultaten. Unmittelbar, nachdem die Ergebnisse der Bonner Physiker ihm bekannt wurden, schrieb er an einen englischen Wissenschaftler:

Zwei junge Physiker in Bonn haben nun die Rotverschiebung der Spectrallinien bei der Sonne so gut wie sicher nachgewiesen und die Gründe des bisherigen Mißlingens aufgeklärt. ${ }^{45}$

Diese Notiz löste dann in der New York Times sogar des Erscheinen einer Notiz über 'Einstein's Third Victory', verfaßt von dem Autor des ersten englischsprachigen Buches über die Relativitätstheorie, Robert Daniel Carmichael (1879-1967), aus, und auch die Kurzreferate der Aufsätze von Grebe und Bachem waren Ende 1920 durchaus wohlwollend verfaßt. ${ }^{46}$ Eine unverhoffte Unterstützung erfuhr Grebe und Bachems Hinweis auf die Bedeutung möglicher Linienasymmetrien auch durch John William Nicholson (1881-1955) und Thomas Ralph Merton (1888-1969), die im November 1920, angeregt durch Grebes und Bachems Arbeit von 1919 eine Studie über die maximal $\mathrm{zu}$ erwartenden Linienverschiebungen in Abhängigkeit vom Grad der Asymmetrie der Linienprofile vorlegten, woraus hervorging, daß Abweichungen der Größenordnung von $0.05 \AA$ zu erwarten waren. ${ }^{47}$ Andere Physiker waren weniger überzeugt davon, daß das so einfach aussehende Resultat von Bachem und Grebe 'so gut wie sicher' feststehe. Der englische Kenner der ART, Arthur Stanley Eddington (1882-1944), fügte seiner Abhandlung über Raum, Zeit und Schwere, nachdem er von Einstein im Februar 1920 über die Arbeit von Bachem und Grebe informiert worden war, eine Skepsis signalisierende Fußnote bei:

In einer neuen Arbeit behandeln Grebe und Bachem einen Fall, der außerordentlich zugunsten der Einsteinschen Verschiebung spricht, und klären die einander widersprechenden Ergebnisse der meisten Forscher auf. Es wird aber trotzdem immer noch das beste sein, abzuwarten und zuzusehen; daher lasse ich die hier gegebene Darstellung so, wie sie ist. ${ }^{48}$

Über die Hintergründe dieser reservierten Stellungnahme erfahren wir Näheres aus dem Brief, mit dem Eddington sich im März 1920 für die Übersendung der Arbeit Bachem und Grebes durch Einstein an ihn bedankte. Zwar sähen die Thesen der

\footnotetext{
${ }^{45}$ Veröffentlicht von Robert W. Lawson, am 29. Januar 1920 in Nature, S. 565. Analog schrieb Einstein an Eddington am 2. Februar 1920: 'In der Frage der Rot-Verschiebung der Spektralen bei der Sonne haben zwei junge Physiker, Grebe und Bachem, in Bonn einen bemerkenswerten Fortschritt erziclt. [...]. Bei Weglassung der verunreinigten Linien finden sie einen mit der Theorie sehr befriedigend übereinstimmenden Wert für die Rotverschiebung' (CPAE, Sign. 9 271).

${ }^{46}$ Siehe Carmichael [1920] bzw. Uhler [1920].

${ }^{47}$ Siche Nicholson und Merton [1920/21] S. 263: 'Thus, though a line may be so narrow that no trace of asymmetry can be detected, it may nevertheless show a displacement which might in certain cases lead to entirely wrong conclusions'.

${ }^{48}$ Eddington [1920/23] S. 134.
} 
Bonner Physiker überzeugend aus, doch stünden sie in Widerspruch zu neuesten Resultaten St. Johns, und in Anbetracht der sich einander gegenüberstehenden Lager innerhalb der Experimentalphysiker wagte Eddington keine Entscheidung zu fällen:

The [Bachem/Grebe] results are very interesting; and look convincing although I am scarcely qualified to judge the questions involved.

I hear that St. John has been making further researches, with magnesium and other lines, still getting a zero result; so I expect that for some time to come spectroscopists will be divided as to what the result really is. ${ }^{49}$

$\mathrm{Zu}$ einfach erschien die Bachem/Grebesche Lösung des Problems dem Manchester Experimentator W. Geoffrey Duffield, der seinen Übersichtartikel 1920 um ein Addendum erweiterte, in dem er auf die Komplexität des Problems, insb. auf die von den Bonner Physikern außer Betracht gelassenen eventuellen Doppler-Effekte, hinwies:

In view of the probable existence of solar currents, I doubt whether the discovery of the full predicted displacement would be conclusive evidence in its [Einstein's ART] favour; the whole problem is inseparable from an examination of the solar circulation $[\ldots]$ in any case, the interpretation cannot be as simple as that given by Grebe and Bachem. ${ }^{50}$

Unverhohlene Skepsis spricht auch aus dem Kommentar, mit dem der Experte für Spektroskopie Charles Edward St. John ${ }^{51}$ (1857-1935) in der Fachzeitschrift Observatory reagierte, als er den Brief Einsteins über Grebe und Bachem in Nature gelesen hatte:

It is, of course, recognized that in a personal letter one may express a more positive opinion than in a note intended for publication; but inasmuch as this statement by Prof. Einstein has been published, it is desirable to call attention to some points relative to the observations by the Bonn physicists [...] that cause solar physicists to hesitate to accept Prof. Einstein's conclusion as to the completeness of the demonstration. [...]. The purpose of this note is not to show that the predicted Einstein displacement is not present in the solar spectrum, but to call attention to some considerations that, in the minds of solar physicists, make it doubtful that the proof in its favour is as complete, and the explanation of previous failure to find it is as convincing, as the brilliant author of the equivalence hypothesis finds them. ${ }^{52}$

Unter diesen Kritikpunkten waren u.a. Verweise auf etliche mögliche Fehlerquellen, sowohl in der Apparatur wie auch in der Auswertungstechnik von Grebe und Bachem, die hier kurz aufgelistet werden sollen, weil sie zeigen, in wie weitem Maße die zunächst so plausibel und stimmig aussehenden Resultate noch kontrovers waren, wie viele implizit gemachte Voraussetzungen in der Arbeit von Grebe u. Bachem noch

\footnotetext{
${ }^{49}$ A. S. Eddington an Einstein, 15. März 1920, CPAE, Sign. 9273.

${ }^{50}$ Duffield [1920] S. 272.

${ }^{51}$ Zu St. John vgl. Hentschel [1990].

${ }^{52}$ St. John [1920]b S. 260.
} 
anfechtbar waren, oder noch anders gewendet, wie wenig transparent ihre Resultate für die astrophysikalische community um 1920/21 waren:

(1) die nicht ausreichende Auflösung des Spektrographs, da in der Cyanbande ungewöhnlich hohe Liniendichten (im Durchschnitt 10 Linien $/ \AA$ ) vorlägen, die bei geringer Dispersion zu systematischen Fehlern führten, ${ }^{53}$

(2) die Komplikationen bei apparativer Einbeziehung des Heliostaten, insb. was die Präzision der Winkelablenkungsmessungen angeht,

(3) mangelnde Vorsichtsmaßnahmen für die Ausschließung von Doppler-RVEffekten, die durch die Sonnenrotation induziert werden - - das gesamte Sonnenbild hatte in der Apparatur Grebe/Bachems nur einen Durchmesser von $5 \mathrm{~cm}$, so daß bei Verschicbung des Ursprungspunktes von nur $1 \mathrm{~mm}$ ein Fehler von bis $\mathrm{zu} 0.1 \mathrm{~km} / \mathrm{sec}$ entstünde, was schon ein Drittel der gemessenen Gesamtbeträge ausmachte,

(4) die nicht-synchrone Herstellung des Vergleichsspektrums, wodurch Temperatur- und Justierungsschwankungen nicht ausgeschlossen werden könnten, die in anderen Fällen bereits als faktische Ursache unrichtiger Messungen nachgewiesen worden seien (St. John nannte hier selbstkritisch frühere Messungen des Mt. Wilson Observatories, also seiner eigenen Institution),

(5) die Unzulässigkeit des Schlusses von den Linienprofilen der im Labor erzeugten Emissionslinien auf die Linienprofile der Absorptionslinien, da letztere durch den umgebenden Dampf beeinflußt werden. Dessen Strahlungsabsorption variere mit der Schichttiefe und deren jeweiliger Temperatur, wodurch i.a. wieder asymmetrische Fraunhofer-Linienprofile entstünden,

(6) die Möglichkeit, daß einige der scheinbaren Linienprofilasymmetrien schlicht durch kleine Ungenauigkeiten im Rowland-Gitter künstlich erzeugt worden seien. Der Test, mit dem dies hätte ausgeschlossen werden können, nämlich das Gitter umzudrehen, und dann zu sehen, ob die Asymmetrien nunmehr gespiegelt auftreten, wurde von Grebe u. Bachem nicht gemacht.

Eine vergleichbare Auflistung von insb. 12 möglichen Schwachstellen veröffentlichte 1922 auch Ludwig Carl Richard Glaser (geb. 1889), ${ }^{54}$ der seit 1920 u.a. in Disputen mit M. v. Laue und E. F. Freundlich nachzuweisen versucht hatte, daß die ART entgegen der seit 1919 weitverbreiteten Auffassung empirisch unhaltbar sei, ${ }^{55}$ und auch schon frühere Arbeiten Grebes skeptisch beurteilt hatte. ${ }^{56}$ Am 2. Sept. 1920 war Glaser nach Weyland der zweite Redner der Arbeitsgemeinschaft deutscher Naturforscher für Rassereinheit der Wissenschaft in der Berliner Philharmonie. Nach dem Bericht über diese Veranstaltung, den Max von Laue (1879-1960) zwei Tage später in die Presse gab, setzte sich Glaser in diesem Vortrag durchaus ernsthaft mit den bisherigen Messungen zur GRV, insb. auch von Bachem und Grebe, auseinander. Diesen Bonner Untersuchungen warf Glaser vor, daß sie auf Messungen mit herkömmlichen Gittern basierten,

\footnotetext{
${ }^{53}$ Ein Beleg dafür waren St. John die von Bachem u. Grebe konstatierten 'erheblichen Schwankungen für die gleiche Linie' je nach Intensität der Belichtung der Photoplatte.

${ }^{54}$ Studium an der TU und Universität Berlin sowie am Imperial College, London, bis 1914; 1916 Dr. ing. an der TU Breslau, seit 1915 Betriebsleiter der Firma Krupp in der Abt. Friedrich Alfred Hütte, seit 1921 Priv. doz. an der Universität Würzburg.

${ }^{55}$ Glaser [1922] - vgl. weiter unten angeführtes Zitat.

${ }^{36} \mathrm{Vgl}$. die Diskussionsbemerkung Glasers zu Grebe/Konen [1921] S. 549: 'Die Versuche sind jedenfalls mit größter Vorsicht zu behandeln'.
} 
welche 'nicht hinreichend fehlerfrei [seien], um diese Untersuchung zu ermöglichen'. ${ }^{7}$ Max von Laue, ein sicherlich jedweder antirelativistischer Vorurteile unverdächtiger Zeuge, bestätigte Glasers Einwänden durchaus ihre Stichhaltigkeit: in der Tat könnten Gitterfehler zu störenden 'Schleiern' führen, die die spektralanalytischen Hochpräzisionsmessungen verfälschen könnten. Doch Glaser habe dies umgekehrt keineswegs beweisen können, und deshalb hielt von Laue die Glasersche Gesamtbewertung der Messungen von Grebe und Bachem für verfehlt:

Diese beim Bonner Apparat auftretenden, bei geeigneten Anordnungen aber fehlenden Schleier sind es nach Glaser, welche Grebe und Bachem zur Ausscheidung der Mehrzahl der bisher untersuchten Linien veranlaßt haben. Glaser hält demgegenüber die älteren Untersuchungen für maßgebend und schloß mit den Worten, er glaube auch die Anhänger der Relativitätstheorie überzeugt zu haben, daß sie von der Rotverschiebung der Spektrallinien nichts mehr zu hoffen hätten.

Darin zeigt sich nun wieder die einseitige Parteinahme dieses sonst nicht schlechten Vortrages. [...]. Der richtige Schluß aus dem vorliegenden Beobachtungsmaterial wäre für einen sehr skeptischen Beurteiler doch wohl der gewesen: die älteren Untersuchungen sind durch Grebe und Bachem in ihrer Bedeutung zweifelhaft gemacht. Deren eigene Untersuchungen sind bisher von anderer Seite nicht nachgeprüft. Also ist die ganze Frage noch in der Schwebe. ${ }^{58}$

Mit dieser autoritativen These des Autors des ersten Lehrbuches über die Relativitätstheorie (1911) war die Diskussion um die Haltbarkeit der Resultate von Bachem und Grebe noch weiterhin offengehalten. Zwischenzeitlich hatte sich auch Einsteins eigene Einschätzung der Resultate von Bachem und Grebe gewandelt, wovon freilich die Öffentlichkeit einstweilen nichts erfuhr. Aus einer schriftlichen Zusammenfassung einer Unterhaltung, die Willem Henri Julius (1860-1925) am 7. Mai mit Einstein hatte, erfahren wir näheres darüber:

Der Schluss[,] zu welchem Grebe und Bachem gelangen, schien uns nicht genügend begründet; denn erstens ist das Mittel $0.56 \mathrm{~km} / \mathrm{sec}$ merklich kleiner als der theoretische Wert 0.63 und fällt sogar auf 0.475 herab, wenn man die eine Linie 3866.960 (die in der früheren Arbeit als unbrauchbar gekennzeichnet wurde) fortlässt; und zweitens wurde nicht gezeigt, dass es ausgeschlossen sei, die beobachteten Verschiebungen als das Resultat der Zusammenwirkung mehrerer Ursachen zu deuten. Mögliche photographische Effekte und Beurteilungsfehler wurden zwar von G. u. B. in Betracht gezogen; aber sogar bei einer alleinstehenden Linie können Druck-, Doppler-Effekt oder anomale Dispersion die Gravitationsverschiebung fälschen. Man kann nicht daran zweifeln, dass verschiedene Ursachen gleicher Grössenordnung mitspielen. ${ }^{59}$

Die starke Abhängigkeit des numerischen Resultats von der Einbeziehung oder NichtEinbeziehung nur einer Linie, die Einstein und Julius bemerkt hatten, spiegelt zunächst nur die schlechte Statistik wieder, die sich Bachem und Grebe durch die rigide Auswahl eingebrockt hatten. Schwerwiegender hingegen war der grundsätzliche Einwand, daß es ihnen nicht gelungen war nachzuweisen, daß die registrierten Verschiebungen nicht

\footnotetext{
${ }^{57}$ Von Laue [1920].

${ }^{58}$ Ibid.

${ }^{59}$ Julius an Einstein, 8. Mai 1920; CPAE, Sign. 14201.
} 
doch von einer Überlagerung mehrerer anderer Effekte erzeugt worden sein könnten-dies wäre in der Tat sehr schwierig gewesen. Doch solange dieser Nachweis ausstand, war ihr Resultat keineswegs der von ihnen erhoffte schlagende Beweis für das Vorliegen der GRV. Wenn nun (im Mai 1920) schon bei Einstein selbst leise Zweifel an der Beweiskräftigkeit der Argumentation von Grebe und Bachem aufgekommen waren, wie mußten da erst die Gegner seiner Theorie denken?

Die Anhänger der von der RT ins Abseits gestellten Äthermodelle klassischer Prägung machten sich nicht so viel Mühe mit der Begründung ihrer skeptischen Haltung gegenüber den Resultaten Bachems und Grebes. Zumeist reichte ihnen schon der bloße Hinweis auf das durch die strengen Selektionskriterien arg zusammengeschrumpfte Datenmaterial. Emil Wiechert etwa, der in diesen Jahren die Gravitation als elektrodynamische Erscheinung aufzufassen suchte, schrieb über Bachem und Grebes Thesen 1920: 'Ehe man sich entschließen könnte, den Verfassern zu folgen, wird man zum mindesten abwarten müssen, bis das Beobachtungsmaterial, welches sie so sehr verkleinert haben, wieder umfangreicher geworden sein wird.' ${ }^{30}$ Mit der späteren Arbeit Grebes an immerhin über 100 Linien entfiel dieser allzu einfache Einwand, so daß die spätere Diskussion um die Richtigkeit der Resultate Bachem und Grebes dann ganz dem kleinen Kreis der Experten, meist mit eigener Erfahrung in spektroskopischen Präzisionsmessungen, vorbehalten blieb.

Im Jahr 1921 fügte Raymond Thayer Birge (geb. 1887) von der University of California noch einen weiteren Kritikpunkt hinzu. In Weiterführung der Arbeiten von Deslandres [1894] war Birge zu Serienformeln für die 10 Doublett-Serien der Cyanbande gekommen, die es ihm gestatten, ihre 'theoretische Position' zu berechnen. Dieser von ihm zusätzlich eingebrachte theoretische Hintergrund, der zurückverweist auf die Phase der Spektroskopie um 1890, als Balmer, Kayser u. Runge, Deslandres u.a. alle auf der Suche nach Serienformeln für die Lage der Linien im Spektrum waren, ermöglichte es Birge, von vielen der von Bachem und Grebe aufgrund ihrer mikrophotometrischen Aufnahmen der Bogen-Emissionslinien für einzeln und schaft gehaltenen Linien nachzuweisen, daß diese de facto aus mehreren nahe beieinanderliegenden Komponenten zusammengesetzt sind. Weil die relativen Intensitäten, mit denen die Komponenten in den Linienkomplexen auftreten, von den physikalischen Bedingungen abhängen, unter denen sie entstehen, war für diese nicht mehr gesichert, daß, auch wenn Grebe/Bachems Aufnahmen der Bogen-Emissionslinien sie als symmetrisch auswiesen, die entsprechenden Absorptionslinien dies auch noch waren. ${ }^{61}$ Von den 11 Linien, die Grebe und Bachem aus ihren 36 Kandidaten

Tabelle 2. Mittlere RV nach Birge [1921], ausgedrückt in Äquivalentgeschwindigkeiten der Konvektionsströme bei Dopplereffekt.

\begin{tabular}{ccccc}
\hline Linie (A) & Grebe/Bachem & Schwarzschild & St. John & Evershed/Royds \\
\hline & 1920 & 1914 & 1917 & 1914 \\
3874.504 & $0.58 \mathrm{~km} / \mathrm{s}$ & $0.45 \mathrm{~km} / \mathrm{s}$ & - & - \\
3858.822 & $0.42 \mathrm{~km} / \mathrm{s}$ & $0.39 \mathrm{~km} / \mathrm{s}$ & $0.40(0.46)$ & - \\
\hline
\end{tabular}

\footnotetext{
${ }^{60}$ Wiechert [1920] S. 330; analog Lenard [1918/21] S. 43 f,

${ }^{61}$ Birge [1921] S. 369: 'It is known that the relative intensity of certain series in the 3883 band changes with physical conditions, and there is evidence that their relative intensity in the sun is different from that in the ordinary carbon arc. Hence any apparent "line" which is really a complex is entirely unsuitable for the detection and measurement of so small a shift as that predicted by Einstein'.
} 
ausgewählt hatten, blieben nach Birges Analyse nur noch zwei [!], die tatsächlich einzelne, scharfe, symmetrische Linien waren. ${ }^{62}$ Für diese zwei Linien stellte Birge dann die von den verschiedenen Beobachtern jeweils ermittelten Verschiebungen zusammen. Bei Mitnahme des höheren von den beiden über zwei unabhängige Methoden bestimmten Wertes von St. John resultierte somit eine über alle Experimentatoren gemittelte RV von $0.46 \mathrm{~km} / \mathrm{s}$, mit zufriedenstellender Übereinstimmung aller Resultate untereinander, ganz im Gegensatz zu den völlig verschiedenen Konklusionen, zu denen die Gruppen selbst in ihren eigenen Auswertungen gekommen waren. Wenn man zu diesem Mittelwert von $0.46 \mathrm{~km} / \mathrm{s}$ noch die Dopplerkorrektur hinzufüge, die sich ergebe, wenn man mit St. John und Adams eine mittlere Aufstiegsgeschwindigkeit der Gase von $0.12 \mathrm{~km} / \mathrm{s}$ in der Mitte der Sonnenscheibe annehme, ergebe sich als wahrer RV-Effekt: $(0.46+0.12=0.58) \mathrm{km} / \mathrm{s}$, der mit der Einsteinschen Voraussage von $0.634 \mathrm{~km} / \mathrm{s}$ immerhin schon größenordnungsmäßig übereinstimme. Doch daß mit dem mageren Beobachtungsmaterial von nur mehr 2 Linien sicherlich eine so weitreichende theoretische Behauptung wie die der Gravitations-RV nicht getestet werden könne, wußte Birge nur zu gut, wichtiger war ihm denn auch, daß seine Re-Analyse erstmals au einer befriedigenden Übereinstimmung der Resultate verschiedener Beobachter geführt hatte: 'it is worthy of notice that the results of all observers are truly consistent on really good lines' ${ }^{63}$

Gegen den späteren Versuch Grebes, die Materialbasis dadurch wieder zu erhöhen, daß er die älteren Messungen an der Cyanbande durch Henry Augustus Rowland (1848-1901) und dessen Mitarbeiter auswertete, die er als theoretisch unvorbelastet ansah, wandte Birge ein, daß Rowland in seiner Tabelle Sohnen und irdische Vergleichsspektren kunterbunt durcheinandergemischt habe, da ihm ja noch nicht bekannt war, daß es systematische Verschiebungen zwischen beiden gebe. Dadurch aber sei die GRV if real, hopelessly involved in the measurements and can not be definitely extricated by any such method as that recently used by Grebe and Bachem'. ${ }^{64}$

Wissenschaftstheoretisch interessant finde ich Birges Neudeutung der Arbeit von Grebe/Bachem deshalb, weil hier genau das Argument, das schon Bachem und Grebe gegen ihre Vorgänger benutzt hatten, nochmals angewandt wurde. Die Bonner Physiker hatten aus den Linien der anderen Gruppen einige wenige, ihrer Meinung nach einzig brauchbare Linien ausgewählt-nun wählte Birge seinerseits aus Grebe/Bachems Vorauswahl nochmals, unter noch strengeren Standards, eine Teilklasse aus. Bemerkenswert ist, daß unter solch mehrmaliger Selektion des Datenmaterials tatsächlich eine höhere Übereinstimmung der mittleren RV resultierte, da rein statistisch genau das Gegenteil zu erwarten wäre. ${ }^{65}$

Die meisten der im vorigen diskutierten Kritikpunkte St. Johns, Birges und Glasers waren ausgesprochen technischer Art; keiner von ihnen bezog sich direkt auf die in Rede stehende Theorie, die ART, sondern alle entstammten instrumentellen und auswertungstechnischen Erfahrungen, die sie im Laufe ihrer Ausbildung und wissenschaftlichen Tätigkeit an spektroskopischen Experimenten mit völlig anderer Zielsetzung gewonnen hatten. Wohl keiner dieser Einwände hätte von einem NichtExperten ausgesprochen werden können. Solange sich die Experten untereinander so wenig einig waren, wie dies hier Grebe/Bachem und Einstein einerseits, St. John, Birge

\footnotetext{
${ }^{62}$ Genau genommen sind auch diese noch Doubletts, aber von symmetrischem Bau.

${ }^{63}$ Birge [1921] S. 370.

${ }^{64}$ Ibid., (Fußnote 63).

${ }^{65}$ Die mittlere durch zufällige Fehler bedingte Streuung ist umgekehrt proportional zur Wurzel der Gesamtzahl der Meßwerte.
} 
und Glaser andererseits, mußte die breitere Öffentlichkeit sich also eines definitiven Urteils über den Stand der Sache enthalten, Entgegen der SensationsBerichterstattung, die mit der Lichtablenkungsmessung von 1919 verbunden gewesen war, scheint sie dies in Bezug auf Grebe/Bachems Resultat auch getan zu haben, wenigstens sind mir keine Zeitungsnotizen hierüber bekannt geworden. Dies ist jedoch nicht etwa darauf zurückzuführen, daß sich die Wissenschaftler noch zu unsicher über ihre eigenen Resultate waren, als daB sie den Schritt an die breitere Öffentlichkeit gewagt hätten. Im Gegenteil: in einem populären Artikel zu 'Grundlagen und Ergebnissen der Relativitätstheorie' für die Monatsschrift Deutsche Revue erweckte Albert Bachem den Eindruck, als ob ihr Resultat bereits unumstößlich feststehe: 'Einen weiteren Beweis für ihre Wahrheit [gemeint ist die Richtigkeit der RT, K.H.] bildet der von deutschen Physikern erbrachte Nachweis, daß die von der Sonne ausgesandten Lichtwellen um einen geringen Betrag, einige zehntausend-millionstel Millimeter von entsprechenden irdischen Lichtwellen abweichen'. ${ }^{66}$ Das stolze Herausstellen der deutschen Nationalität der Experimentatoren, die diesen angeblichen 'Beweis' erbrachten muß man auch sehen als bewußten Kontrapunkt gegen die ebenfalls oft betonte englische Nationalität der Mitglieder der Expeditionen nach Principe, die die Lichtablenkung maßen. Es ist aber sehr wohl möglich, daß durch diesen Versuch der Vereinnahmung der GRV sozusagen als 'deutschen' Effekt den noch unentschlossenen, häufig deutschnational eingestellten Physikern der Weg zur Akzeptierung der ART bereitet werden sollte.

An der Abfolge der Äußerungen von Arnold Sommerfeld (1868-1951) jedenfalls läßt sich zwischen 1919 und 1921 eine zunehmende Akzeptanz der ART gerade unter Hinweis auf die jeweils neuesten Ergebnisse Bachem/Grebes feststellen. In seinen Anmerkungen für die Aufsatzsammlung Das Relativitätsprinzip mit Aufsätzen von Lorentz, Einstein, Minkowski und Weyl vermerkte er vor 1920 noch lakonisch: 'Die Rotverschiebung der Spektrallinien durch das Gravitationsfeld der Sonne oder anderer Gestirne von großer Masse, die die Theorie verlangt, wird aber durch die besten Messungen des Mount-Wilson-Observatoriums widerlegt' ${ }^{67} 1920$ wurde dem von Sommerfeld, noch immer deutlich distanziert, hinzugesetzt: 'auch die Aussichten für den Nachweis der Rotverschiebung haben sich inzwischen merklich gebessert'. ${ }^{68}$ Nach den statistisch besser abgesicherten Untersuchungen Grebes schrieb Sommerfeld dann 1921, sowohl im Archiv für Elektrotechnik als auch in den Süddeutschen Monatsheften:

Diese Rotverschiebung steht zur Zeit im Mittelpunkt des Interesses. Von Schwarzschild wurde sie an gewissen Stickstofflinien des Sonnenspektrums nachgewiesen, aber in einem kleineren Betrage als aus der Einsteinschen Berechnung folgt. Merkwürdigerweise hat sich mit den vorzüglichen Mitteln des Mount-Wilson-Observatoriums fast keine Spur des Effektes ergeben. Dagegen haben kürzlich die Herren Grebe und Bachem in Bonn an denselben Stickstofflinien unter planmäßiger Auswahl von 6 ungestörten und Ausschaltung aller durch Störungen verdächtigen Linien die Rotverschiebung quantitative bestätigt. Eine weitere Überraschung brachte die diesjährige Nauheimer Naturforscherversammlung. Indem dieselben Herren eine Statistik von 100

\footnotetext{
${ }^{66}$ Bachem [1921] S. 21.

${ }^{67}$ Sommerfeld (Hrsg.) [1923].

${ }^{68}$ Unter Verweis auf die beiden frühen Arbeiten Grebe und Bachem [1919], [1920].
} 
Stickstofflinien, gestörten und ungestörten, durchführten, wurden sie abermals im wesentlichen auf den Einsteinschen Wert der Rotverschiebung geführt. Wir haben also allen Grund anzunehmen, daß die weitere Verfolgung auch dieses schwierigen Problems mit einem vollen Siege der Einsteinschen Prophezeiung endigen wird. ${ }^{69}$

Demgegenüber spürt man besonders in Glasers Arbeit sehr deutlich das Bemühen, die noch unentschlossene Öffentlichkeit nicht in die Meinung verfallen zu lassen, daß die ART bereits endgültig bestätigt sei, obwohl eben dieser Mythos sich seit 1919 in einer zunehmenden Flut populärer Schriften zur $\mathrm{RT}^{70}$ niederschlug und selbst die noch zögernden Experten sich von Ergebnissen wie denen von Bachem und Grebe beeindrucken ließen (wie eben am Beispiel Sommerfelds gezeigt). Direkt gegen Sommerfelds oben zitierte Äußerung schrieb Glaser 1922: 'Bei sorgfältiger Prüfung wird man sich daher kaum dem Sommerfeldschen Urteil anschließen können, daß die Arbeiten von Grebe und Bachem, insonderheit die von Grebe vorgetragene Statistik der Verschiebung Fraunhoferscher Linien, eine 'Überraschung' sind." Ebenso energisch beantwortete Glaser $1920 \mathrm{v}$. Laues Zeitungsartikel über eine angebliche Bestätigung der ART mit einer gegenlautenden Notiz. Von der Voreiligkeit der Schlüsse der beiden Bonner Physiker überzeugt, suchte er in ihrer Arbeit nach möglichen Schwachstellen, darum auch veröffentlichte Glaser eigene Übersichten zum Stand der Prüfung der ART. ${ }^{72}$

Als Freundlich am 1. März 1920 in der Sitzung des Vereins zur Beförderung des Gewerbefleißes $^{73}$ einen Vortrag hielt, in dem die ART als der Endpunkt einer 'Entwicklung des physikalischen Weltbildes' dargestellt wurde, widersprach Glaser unter Verweis auf die ausstehende experimentelle Prüfung der GRV heftig, ${ }^{74}$ woraufhin Freundlich konterte:

Zwei Bonner Physikern, Grebe und Bachem, ist es gelungen, nachzuweisen, daß in den Messungen der Amerikaner eine Fehlerquelle steckt, so daß heute wieder die Frage offen ist. Jedenfalls sind die Messungen der Amerikaner nicht richtig gewesen, wenngleich die Fehlerquelle noch nicht ganz aufgeklärt ist. Die Struktur der Linien ist nicht die gleiche, und sie zu untersuchen, wird wohl noch längere Zeit in Anspruch nehmen. ${ }^{75}$

Obgleich sich Freundlich bei dieser Berufung auf die Grebe/Bachem [1919], [1920] darauf beschränkte, sie als ein Argument gegen den Widerlegungsversuch St. Johns von 1917 zu verwerten, also nicht etwa positiv als Beweis der Richtigkeit der GRV wertete,

${ }^{69}$ Sommerfeld [1921]a S. 398.

${ }^{70}$ Vgl. dazu Hentschel [1989] Kap. 2.

${ }^{71}$ Glaser [1922] S. 102.

721920 in Glasers Annalen für Gewerbe und Bauwesen, weil die Standard-Zeitschriften deren Publikation wohl verweigert hatten, 1923 in dem vom Antirelativisten Stark herausgegebenen Jahrbuch für Radioaktivität und Elektronik.

${ }^{73}$ Am Rande sei bemerkt, daß Vorträge wie dieser zeigen, wie weit die Wellen schlugen, die die ART nach 1919 erzeugte- das Interesse an der ART ging weit über die gesellschaftlichen Gruppen hinaus, die normalerweise an wissenschaftlichen Entwicklungen Anteil nahmen.

${ }^{74}$ Siehe die Diskussion hinter Freundlich [1920]b S. 58.

${ }^{75}$ Freundlich [1920]b S. $58 \mathrm{f}$. Mit den Messungen der Amerikaner sind die Arbeiten von St. John [1917]ff. gemeint - vgl. dazu Hentschel [1990]. 
so entzündete doch schon dieser eingeschränkte Verweis auf die Bonner Untersuchungen Glasers Zorn, dem er in einer 'Mitteilung an die Schriftleitung' Ausdruck verlieh.

Wenn neuerdings in Bonn von den Physikern Grebe und Bachem [...] mit unzureichenden Hilfsmitteln, die den Vergleich mit den bisherigen oben erwähnten Untersuchungen [St. Johns, K.H.] kaum vertragen, versucht wird, durch Anbringung von sehr zweifelhaften Verbesserungen an den bisher veröffentlichten sowie an ihren eigenen gleichfalls negativen Versuchsergebnissen 'der Existenz des Einsteineffektes ein hohes Maß von Wahrscheinlichkeit' zuzuweisen, so muß, abgesehen von einigen bedenklichen Stellen der experimentellen Durchführung, auf die an anderer Stelle zurückzukommen sein wird, das Verfahren einige unbequeme, mit sehr großen Hilfsmitteln ausgeführte Messungen wie die von St. John von der Diskussion stillschweigend auszuschließen oder nicht genügend zu würdigen, als wissenschaftliche in höchstem Maße bedenklich erscheinen. ${ }^{76}$

Eigentlich wollte Glaser schon auf der Naturforscherversammlung in Bad Nauheim 1920 seine Kritik an den bis dato vorliegenden Arbeiten Bachems und Grebes vortragen, doch kam es dazu nicht. Stark gegenüber stellte er es so dar, daß er aufgrund eines Trauerfalls in seiner Familie an einer Teilnahme verhindert gewesen sei, ${ }^{77}$ doch erscheint mir dies eher unplausibel - hätte sich Glaser die Gelegenheit nehmen lassen, seine Einwände zu erheben, wenn er sie wirklich für so stichhaltig erachtete? Möglicherweise fürchtete er, daß seine etwas aus dem Stegreif entwickelten Einwände ihn vor der sachkundigen Zuhörerschaft eher blamiert hätten, als daß sie akzeptiert worden wären. Doch dies bleibt Spekulation, solange ich nicht mehr Dokumente aus der Feder Glasers aufgefunden habe. ${ }^{78}$ Die hier angekündige Detailkritik Glasers zur experimentellen Durchführung erschien 1922, gefolgt von einem ebenfalls vernichtenden Überblick zum Gesamtstand der Experimente zur GRV im Jahr 1923. Inhaltlich folgte sie weitgehend den Einwänden Birges, so daß sich ein erneutes Referat hier erübrigt. Als eine Art Resumé seiner Kritik an dem neuen Bestätigungsversuch der ART durch Grebe und Bachem stellte Glaser 1922 fest, daß die von den bisherigen Resultaten so auffällig 'abweichenden Ergebnisse Grebes und Bachems auf unzulängliche Versuchshilfsmittel und unzureichende Ausführung der Versuche zurückzuführen sind. Überdies genügen andere physikalische Gründe zur Erklärung der in geringem Maße vorhandenen Rotverschiebung der Fraunhoferschen Linien und machen daher die Erklärung durch die Einsteinsche Relativitätstheorie entbehrlich. Ihr Nichtvorhandensein in dem von Einstein errechneten Betrage ist für die auch in anderer Beziehung haltlos gewordene Relativitätstheorie vernichtend, da nach Einsteins eigenen Worten die allgemeine Relativitätstheorie bei Nichtvorhandensein der Rotverschiebung unhaltbar ist. ${ }^{, 79}$

\footnotetext{
${ }^{76}$ Glaser [1920]b S. 105.

${ }^{77} \mathrm{Vgl}$. das Telegramm Glasers an Stark, 24. September 1920, SPK, Nachlaß Stark, Mappe Glaser, Nr. 12: 'muß ich mir im Hinblick auf die Aufregungen, die mir der Tod meiner Schwester bereitet hat, versagen, die Ergebnisse meiner wissenschaftlichen Arbeit dort vorzutragen, zumal die auf demagogische Wirkung berechneten Angriffe von einer Seite erfolgen, die sachlich mir in dem wissenschaftlichen Streit um die Relativitätstheorie unbekannt ist'.

${ }^{78}$ Am Rande sei erwähnt, daß Glaser während der NS-Zeit ganz im Stile der Autoren der 'Deutschen Physik' antisemitische Hetze betrieb-siehe Glaser [1939].

${ }^{79}$ Glaser [1922] S. $101 \mathrm{f}$.
} 
Hier wird die GRV, von Einstein selbst durch sein kompromißloses Insistieren auf der RV als unabdinglicher Konsequenz der ART zum experimentun crucis stilisiert, vom Antirelativisten Glaser zum Kronzeugen gegen die ART erklärt. Die Bedeutung, die gerade dieses Experiment nach 1919 dadurch sowohl für die Pro- als auch für die Anti-Relativisten erlangte, erklärt die Heftigkeit, mit der zwischen 1919 und 1924 innerhalb der scientific community um die 'richtige' Deutung der vorhandenen Daten gestritten wurde. Sie erklärt auch die Intensität, mit der in diesem Zeitraum nach neuen Wegen gesucht wurde, auf denen man unanfechtbare finden könnte. Leonhard Grebes und Albert Bachems Versuche waren einer der dabei beschrittenen Wege; die kritische Rezeption ihrer Thesen zeigt, daß es noch nicht der Königsweg war.

\section{Das weitere Schicksal Bachems und Grebes}

Beide Bonner Physiker wandten sich denn auch schon seit Anfang der 20er Jahre mehr und mehr dem Feld der physikalischen Medizin zu. Bachem wurde 1920 Privatdozent für medizinische Physik in Frankfurt am Main und ging 1924 in die USA, wo er von 1924-1954 an der University of Illinois, Chicago, wiederum über medizinische Anwendungen der Physik (insb. von Röntgenstrahlen und UV-Licht) lehrte. ${ }^{80}$ Außer einer knappen Notiz in der New York Times vom 12. April 1957, einen Tag nach seinem Tod, scheinen jedoch keine weiteren Nachrufe über Bachem veröffentlicht worden zu sein, was auf eine gewisse fachliche Isolierung schließen läßt. Auch Grebe wandte sich dem Feld der medizinischen Anwendungen der Physik zu, allerdings ohne den Weg ins Ausland. Seit 1921 bis zu seiner Emeritierung 1952 war er Professor an der Universität Bonn, und ab 1922 darüber hinaus auch Leiter des Röntgen-Forschungsinstituts der Universität Bonn. ${ }^{81}$

Insbesondere der Lebensweg Albert Bachems, dem es nicht gelang, in Deutschland im akademischen System Fuß zu fassen, ist typisch für das Schicksal vieler Mitarbeiter Einsteins, die aufgrund ihrer offenen Befürwortung der RT den gleichen Anfeindungen des großteils konservativ eingestellten community wie Einstein selbst ausgesetzt waren, ohne daß ihnen in gleichem Maße die Möglichkeiten offenstanden, sich zur Wehr zu setzen:

If Einstein did not share the neglect that his collaborators suffered, it was only because, by 1909, the latitude and genius of his physics made it extraordinarily difficult for other physicists to damage his reputation by personal vindictiveness. His young collaborators became victims of the rejection of his thought by the Wilhelmian physical science establishment. ${ }^{82}$

Mit anderen Worten: Bachem bezog die geistigen Prügel, die eigentlich Einstein und seiner verfemten Theorie galten. ${ }^{83}$ Grebe hingegen fand eine akademische Nische, die jedoch mit seinen früheren spektroskopischen Studien zur GRV bezeichnenderweise nichts zu tun hatte, d.h. er festigte seine Stellung im akademischen System nur durch radikale Umorientierung seiner Forschungsinteressen.

\footnotetext{
${ }^{80}$ Man vergleiche neben seinen hier nicht angeführten Fachpublikationen in diesem Feld auch den populären Artikel in der New York Times, 16. November 1930, Sect. IX, S. 4: 'Saving man from civilization. The high task of medicine'. Im Archiv der University of Illinois, Chicago hat sich kein NachlaB Bachems erhalten.

${ }^{81}$ Biographische Daten laut Poggendorff, Bd. V-vilb; vgl. auch die Angaben über Ehrenzeichen im Eintrag des Reichshandbuches der deutschen Gesellschaft [1930].

${ }^{82}$ Pyenson [1985] S. 238 (im Hinblick auf Freundlich, Laub und Ritz).

${ }^{83}$ Vgl. dazu Hentschel [o.J.]
} 
Als Einstein im Juni 1954 dem aus der Lehrtätigkeit an der Universität von Chicago ausscheidenden Albert Bachem unter Verweis darauf, daß er selbst schon seit zehn Jahren nicht mehr aktiv sei, versicherte, daß 'man an dieser beschaulichen Situation sehr Wohl gefallen finden kann', so war dies im Hinblick auf seine eigene AußenseiterStellung in Princeton eine eigenartige Mischung aus Witz und Resignation. So beeilte er sich hinzuzufügen, daß ihm Bachems Arbeiten über die Rotverschiebung von 191922 'noch in frischem Gedächtnis' seien, ${ }^{84}$ doch wußten sie beide genau, daß dieser Gedächtnis-Eintrag unter der Rubrik 'ehrgeizige, aber gescheiterte Versuche der Bestätigung der GRV-Voraussage der ART' erfolgt war.

\section{Danksagungen}

Diese Studie entstand während eines einjährigen Aufenthaltes an der TU Berlin als Rathenau-Stipendiat des Berliner Verbundes für Wissenschaftsgeschichte. Mein Dank gilt allen, die mich dort mit Rat und Tat unterstützten. Für Kritik an einer früheren Fassung dieses Papers danke ich den Herrn Professor Dr. Karl Hufbauer, University of California Irvine und Professor Dr. Fritz Krafft, Universität Marburg sowie Miss Ann Lehar, Boston. Ferner danke ich Herrn Dr. Henning vom Archiv der Max-PlanckGesellschaft, Berlin-Dahlem, und den Editoren der Collected Papers of Albert Einstein (CPAE) für die Gewährung von Einsicht in die dortigen Bestände. Für die Erlaubnis zur wörtlichen Wiedergabe der Dokumente von und über Bachem und Grebe danke ich dem Archiv der Max-Planck-Gesellschaft (AMPG), dem Zentralarchiv der Akademie der Wissenschaften der ehemaligen DDR (AAW), der Stiftung Preußischer Kulturbesitz, Berlin (SPK), und der Hebrew University of Jerusalem als Inhaberin der Rechte an unveröffentlichten Einstein-Dokumenten.

${ }^{84}$ Einstein an Bachem, Juni 1954, CPAE, Sign. 6 051-1.

\section{Literaturverzeichnis}

Bachem, Albert 'Das Bogenspektrum des Zirkons', Zeitschrift für wissenschaftliche Photographie, $8(1910), 316-32$.

Bachem, A. 'Magnetoptische Untersuchungen an der Stickstoffbande 3883', Zeitschrift für Physik, 3 (1920), 372-88.

Bachem, A. 'Grundlagen und Ergebnisse der Relativitätstheorie', Deutsche Revue, 46, 2 (1921), $11-23$.

Bachem, A. 'Saving man from civilization. The high task of medicine. Thrust into an environment in many ways injurious, the race may hopefully look to scientists to solve this problem', New York Times, 10. November 1930, Sect. IX, 4.

Bachem, A. [Todesnotiz], New York Times 12. April 1957, 25.

Barratt, S. 'The origin of the "cyanogen" bands', Proceedings of the Royal Society London, A 98 (1920), 40-49.

Birge, Raymond Thayer 'The first Deslandres' group of the positive band spectrum of nitrogen, under high dispersion', Astrophysical Journal, 39 (1914), 50-88.

Birge, R. T. 'Notes on the use of the concave grating', Astrophysical Journal, 43 (1916), 81-85 (= Replik auf Grebe [1914]).

Birge, R. T. 'The mathematical structure of band series', Astrophysical Journal, 46(1917), 85-103.

Birge, R. T. 'The mathematical structure of band series', Physical Review, (2) 11 (1918), 136-37; 13 (1919), 360-73.

Birge, R. T. 'The relativity shift of spectral lines', Science, 53 (1921), 368-70 (=Kritik v. Grebe/Bachem [1920]).

Birge, R. T. 'The quantum theory of bound spectra and its application to the determination of temperature', Astrophysical Journal, 55 (1922), 273-90. 
Birge, R. T. 'The 3883 Cyanogen band in the solar spectrum', Astrophysical Journal, 59 (1924), $45-60$.

Carmichael, Robert Daniel 'Einstein's third victory. Red-Displacement of spectral lines regarded as completing proof of relativity theory', New York Times, 28. März 1920, Sect. V, 11.

Duffield, W. G. 'The displacement of spectrum lines and the equivalence hypothesis', Monthly Notices of the Royal Astronomical Society, 80 (1920), 262-72.

Earman, John and Glymour, Clark 'The gravitational redshift as a test of general relativity: history and analysis', Studies in History and Philosophy of Science, 11 (1980), 251-78.

Eberhard, Gustav 'Photographische Photometrie', Handbuch der Astrophysik, 2, Teil 2, (1931) 2, Hälfte, 431-518.

Eddington, Arthur Stanley Raum, Zeit und Schwere. Ein Umriß der allgemeinen Relativitätstheorie (1920-23) (=Dt. Übers. durch W. Gordon, Braunschweig, Vieweg; Die Wissenschaft, Bd. 70).

Elster, J. and Geitel, H. 'Der photoelektrische Effekt am Kalium bei sehr geringen Lichtstärken', Physikalische Zeitschrift, 13 (1912), 468-76.

Elster, J. and Geitel, H. 'Über eine scheinbare photoelektrische Nachwirkung am Kalium und über den Einfluß der Entgasung auf den Photoeffekt an Metallen', Physikalische Zeitschrift, 21 (1920), 361-67.

Evershed, John 'A new method of measuring small displacements of spectrum lines', Kodaikanal Observatory Bulletin, 32 (1913a), 17-25.

Evershed, J. 'A new interpretation of the general displacements of the lines of the solar spectrum towards the red', Kodaikanal Observatory Bulletin, 36 (1913b), 45-53.

Evershed, J. 'On the displacement of the spectrum lines at the sun's limb', Kodaikanal Observatory Bulletin, 39 (1914a), 71-81; Observatory, 27 (1914b), 124-28.

Evershed, J. 'The general shift of Fraunhofer lines towards the red', Observatory, 37 (1914c), 388.

Evershed, J. 'Note on radial movement in sun-spots', Astrophysical Journal, 40 (1914d), 156-58.

Evershed, J. 'The Einstein effect and the eclipse of 1919, May 29', Observatory, 40 (1917), 269-70.

Evershed, J. 'The displacement of Cyanogen bands in the solar spectrum', Observatory, 41 (1918), $371-75$.

Forbes, Eric Gray 'A history of the solar red shift problem', Annals of Science, 17 (1961), 129-64.

Forbes, E. G. 'Freundlich, Erwin Finlay', Dictionary of Scientific Biography, 5 (1972), 181-84.

Freundlich, Erwin F. 'Die Entwicklung des physikalischen Weltbildes bis zur allgemeinen Relativitätstheorie', Die weißen Blätter, 7 (1920a), 174-91; Sitzungsberichte des Vereins zur Förderung des Gewerbefleißes, 99 (1920b), 1 März, 45-59 (mit Disk.; s.a. Replik v. Glaser [1920]d, ibid., Heft 6).

Glaser, Ludwig C. 'Eisen als Bezugsspektrum für Wellenlängenbestimmungen', Zeitschrift für wissenschaftliche Photographie, 11 (1912), 375-80; 'Druckfehlerberichtigung', ibid, 12 (1912), 308.

Glaser, L. C. 'Zur Erörterung über die Relativitätstheorie', Tägliche Rundschau, 16. August (1920a), Nr. 180.

Glaser, L. C. 'Versuche zur Bestätigung der Relativitätstheorie', Glasers Annalen für Gewerbe und Bauwesen, 87 (1920b), 29-33; 88 (1920c), 30-33, 42-43; als Separatum: Berlin, 1920 (=Schriften aus dem Verlag der Arbeitsgemeinschaft Deutscher Naturforscher zur Erhaltung reiner Wissenschaft, Bd. 3).

Glaser, L. C. 'Bemerkungen zu dem im Verein zur Beförderung des Gewerbefleißes am 1. März (1920d) von Herrn Dr. Freundlich gehaltene Vortrage 'Die Entwicklung des physikalischen Weltbildes bis zur allgemeinen Relativitätstheorie", Sitzungsberichte des Vereins zur Beförderung des Gewerbefleißes, 99 (1920), 105-106.

Glaser, L. C. 'Uber die Gravitationsverschiebung der Fraunhoferschen Linien (Bemerkung zu einer gleichnamigen Arbeit von L. Grebe), Physikalische Zeitschrift, 23 (1922), 100-102 (s.a. Erw. v. Grebe [1922]).

Glaser, L. C. 'Die Einsteinsche Relativitätstheorie und die Rotverschiebung der Fraunhoferschen Linien', Jahrbuch der Radioaktivität und Elektronik, 20 (1923), 277-352.

Glaser, L. C. 'Juden in der Physik: jüdische Physik', Zeitschrift für die gesamte Naturwissenschaft, 5 (1939), 272-75.

Goos, Fritz 'Über eine Neukonstruktion des registrierenden Mikrophotometers', Zeitschrift für Instrumentenkunde, 41 (1921), 313-24.

Grebe, Leonhard (Christian Friedrich) 'Über den Einfluß der Temperatur auf eine Rowlandsche Gitteraufstellung', Zeitschrift für wissenschaftliche Photographie, 13 (1914), 264-68 (s.a. Replik v. Birge [1916]). 
Grebe, L. 'Über die Gravitationsverschiebung der Fraunhoferschen Linien', Physikalische Zeitschrift, 21 (1920a), 662-68.

Grebe, L. 'Über ein einfaches Einfadenelektrometer', Zeitschrift für Physik, 3 (1920b), 329.

Grebe, L. 'Sonnengravitation und Rotverschiebung', Zeitschrift für Physik, 4 (1921), 105-109.

Grebe, L. 'Über die Gravitationsverschiebung der Fraunhoferschen Linien (Bemerkung zu vorstehender Notiz des Herrn Dr. Ing. L. C. Glaser)', Physikalische Zeitschrift, 23 (1922), 102 (= Erw. auf Glaser [1922]).

Grebe, L. 'Klassische und moderne Interferenzversuche und Interferenzapparate. Elementare Theorie derselben', Handbuch der Physik, 20 (1928a), 1-34.

Grebe, L. 'Beugung', Handbuch der Physik, 20 (1928b), 35-66.

Grebe, L. 'Weißes Licht. Gesetzmäßigkeiten schwarzer und nichtschwarzer Strahlung', Handbuch der Physik, 20 (1928c), 121-40.

Grebe, L. 'Röntgenspektra', Handbuch der Physik, 21 (1929a), 329-59.

Grebe, L. 'Spektralanalyse im Röntgengebiet', Handbuch der Physik, 21 (1929b), 678-82.

Grebe, L. [Kurzbiographie], Reichshandbuch der Deutschen Gesellschaft, 1 (1930), 586-87.

Grebe, L. and Bachem, A. 'Uber den Einsteineffekt im Gravitationsield der Sonne', Verhandlungen der Deutschen Physikalischen Gesellschaft, 21 (1919), 454-64 (s.a. St. John [1920]b, Schulze [1921]).

Grebe, L. and Bachem, A. 'Über die Einsteinverschiebung im Gravitationsfeld der Sonne', Zeitschrift für Physik, 1 (1920a), 51-54 (s.a. Uhler [1920]).

Grebe, L. and Bachem, A. 'Die Einsteinsche Gravitationsverschiebung im Sonnenspektrum der Stickstoffbande $\lambda=3883$ AE', Zeitschrift für Physik, 2 (1920b), 415-22.

Grebe, L. and Holtz, O. 'Ursprung und Struktur der ultravioletten Wasserdampfbanden $\lambda=3064$ ', Annalen der Physik, (4) 39 (1912), 1043-50.

Grebe, L. and Konen, H. 'Bandenspektra von Isotopen', Physikalische Zeitschrift, 22 (1921), 54649.

Grotian, W. and Runge, C. 'Die sogenannten Cyanbanden', Physikalische Zeitschrift, 15 (1914), $545-48$.

Hartmann, J. 'Apparat und Methode zur photographischen Messung von Flächenhelligkeiten', Zeitschrift für Instrumentenkunde, 19 (1899), 97-103.

Hassenstein, Walter 'Visuelle Photometrie', Handbuch der Astrophysik, 2, Teil 2(1931), 2. Hälfte, 519-746.

Heisenberg, Werner 'Das Kaiser-Wilhelm-Institut für Physik. Geschichte eines Instituts', Jahrbuch der Max-Planck-Gesellschaft für 1971, (1971), 46-89.

Hentschel, Klaus Interpretationen und Fehlinterpretationen der speziellen und allgemeinen Relativitätstheorie durch Zeitgenossen Albert Einsteins, Dissertation, Universität Hamburg, 1989; Basel et al., Birkhäuser, 1990 (=Science Networks, Bd. 6).

Hentschel, K. 'The conversion of St. John', to appear in Science in Context, 1991/92.

Hentschel, K. Der Einstein-Turm, E. F. Freundlich und die Relativitätstheorie (Berlin, Spektrum Akademischer Verlag, 1992).

Hentschel, K. [o.J.] 'The rise of antisemitic argument against Einstein's theories of relativity', unveröffentlichtes Manuskript.

Herrmann, Dieter B. and Hoffmann, Dieter 'Astrofotometrie und Lichttechnik in der 2. Hälfte des 19. Jahrhunderts', NTM-Schriftenreihe, 13 (1976), 94-104 (= Mitteilungen der Archenhold-Sternwarte, Nr. 116).

Jewell, Lewis E. 'The coincidence of solar and metallic lines', Astrophysical Journal, $3(1896), 89$ 113.

Kayser, Heinrich and Eversheim, Paul 'Das physikalische Institut der Universität Bonn', Physikalische Zeitschrift, 14 (1913), 1001-08.

King, Arthur Scott 'Intensity differences in furnace and arc among the component series in band spectra', Astrophysical Journal, 53 (1921), 161-64 (=Contributions from the Mount Wilson Observatory, Nr. 194).

King, A. S. and Koch, Peter Paul 'An application of the registering microphotometer to the study of certain types of laboratory spectra', Astrophysical Journal, 39 (1914), 213-29 (=Contributions from the Mount Wilson Observatory, Nr. 77).

Klüber, Harald v. 'Erwin Finlay-Freundlich', Astronomische Nachrichten, 288 (1965), 281-86.

Koch, Peter Paul 'Über ein registrierendes Mikrophotometer', Annalen der Physik, (4) 39 (1912), $705-51$. 
Koch, P. P. 'Über die Messung der Intensitätsverteilung in Spektrallinien. II. Die Eliminierung des Apparateinflusses und die Deutung der wahren Intensitätsverteilung', Annalen der Physik, (4) 42 (1913), 1-29.

Laue, Max von 'Der Einstein-Effekt im Spektrum', Vossische Zeitung, 4. September 1920, Abendausgabe, Nr. 438; B 208, $2-3$.

Laue, M. v. 'Einsteins Gravitationstheorie bestätigt. Die Rotverschiebung der Spektrallinien auf der Sonne', Vossische Zeitung, 29 April 1924.

Lehmann, Erich 'Eine neue Photometer-Konstruktion', Verhandlungen der Deutschen Physikalischen Gesellschaft, 13 (1911), 335-37.

Lenard, Philipp 'Über Relativitätsprinzip, Äther, Gravitation', Jahrbuch der Radioaktivität und Elektronik, 15 (1918), 117-36; als Separatum: Leipzig, Hirzel, 1. Aufl. 1920; 3. erweit. Aufl. 1921.

Nicholson, John William and Merton, Thomas Ralph 'On the effect of asymmetry on wavelength determination', Proceedings of the Royal Society, London, A 98 (1920-21), 261-63.

Nipperdey, Thomas and Schmugge, Ludwig 50 Jahre Forschungsförderung in Deutschland: ein Abriß der Geschichte der DFG, 1920-1970 (Bonn, DFG, 1970).

Pyenson, Lewis The Young Einstein. The Advent of Relativity (Bristol, Hilger, 1985).

Richter, Steffen 'Das Wirken der Notgemeinschaft der Deutschen Wissenschaft erläutert am Beispiel der Relativitätstheorie in Deutschland 1920-1930', Physikalische Blätter, 27 (1971), 497-504.

Rosenberg, Hans 'Lichtelektrische Photometrie', Handbuch der Astrophysik, 2, Teil I, 1. Hälfte (1929), 380-430.

Rowland, Henry Augustus Preliminary Table of Solar Spectrum Wavelengths (Chicago, University of Chicago Press, 1896).

St. John, Charles Edward 'A search for an Einstein relativity-gravitational effect in the sun', Proceedings of the National Academy of Sciences, 3 (1917a), 450-52.

St. John, C. E. 'The principle of generalized relativity and the displacement of Fraunhofer lines toward the red', Astrophysical Journal, 46 (1917b), 249-69 (=Contributions from the Mount Wilson Observatory, Nr. 138).

St. John, C. E. 'Displacement of solar lines and the Einstein effect', Observatory, 43 (1920a), $158-62$.

St. John, C. E. 'The displacement of solar spectrum lines', Observatory, 43 (1920b), 260-62 (=Komm. zu Einstein [1920] über Grebe/Bachem [1919]f).

Schulze, Franz Arthur 'Die Rotverschiebung im Sonnenspektrum', Zeitschrift für Physik, 5 (1921), 371-73.

Schwarzschild, Karl 'Über die Verschiebung der Banden bei $3883 \AA$ im Sonnenspektrum', Sitzungsberichte der Preußischen Akademie der Wissenschaften, Berlin, (1914), 1201-13.

Sommerfeld, Arnold (Johannes Wilhelm) (Hrsg.) Lorentz-Einstein-Minkowski: Das Relativitätsprinzip (Leipzig, Teubner, 1. Aufl. 1913; 5. Aufl. 1923; seither unverändert wiederaufgelegt).

Sommerfeld, A. 'Kurzer Bericht über die allgemeine Relativitätstheorie und ihre Prüfung an der Erfahrung', Archiv für Elektrotechnik, 9 (1921a), 391-99.

Sommerfeld, A. 'Die Relativitätstheorie', Süddeutsche Monatshefte, 18 (1921b), 1268-71.

Uhler, Horace Scudder 'The Einstein displacement of solar lines', American Journal of Science, 200 (1920), 394 (= Ref. v. Grebe u. Bachem [1920]a).

Uhler, H. S. and Patterson, R. A. 'The structure of the third cyanogen band and the associated tails', Astrophysical Journal, 42 (1915), 434-68.

Wiechert, Emil 'Die Gravitation als elektrodynamische Erscheinung', Kurzfassung in Nachrichten der Akademie der Wissenschaften, Göttingen, 1918, 1-8; Annalen der Physik, (4) 63 (1920), 301-89.

Wolfschmidt, Gudrun 'Die Entwicklung der astronomischen Photometrie bis in die 20er Jahre', Deutsches Museum, Wissenschaftliches Jahrbuch für 1989, (1989), 227-71. 